\title{
Risk, Uncertainty and Optimism in Venture Capital Relationships *
}

\author{
Yahel Giat Steven T. Hackman $^{\ddagger}$ and Ajay Subramanian ${ }^{\S}$
}

January 11, 2007

\begin{abstract}
We develop a dynamic, structural model to quantitatively assess the effects of risk, uncertainty and asymmetric beliefs about project quality on the characteristics of venture capital relationships. We estimate the model parameters with data about the distributions of total investments, payoffs, risks and returns of venture capital projects. Entrepreneurial optimism mitigates the agency costs of risk-sharing between venture capitalists (VCs) and entrepreneurs (ENs) by over $20 \%$ and significantly enhances the VC's expected payoffs. The EN optimism premium implied by the data explains the huge discrepancy between the discount rates used by $\mathrm{VCs}(\sim 40 \%)$, which adjust for optimistic payoff projections by ENs, and the average expected return of $\mathrm{VC}$ projects $(\sim 15 \%)$. Consistent with observed contractual structures, the equilibrium dynamic contracts feature both equity-like and debt-like components for the VC and progressive vesting of the EN's stake. The duration, project value and the VC's expected payoff all increase with the project's transient risk but decrease with its intrinsic risk.
\end{abstract}

JEL Classification Codes: G24, D92, D80, G12

\footnotetext{
${ }^{*}$ We thank Anastasia Kartashevea, Zhiyong Liu, Dan Peled, Haim Reisman, Antoine Renucci, Alex Shapiro, Adam Speight, Eric Ulm, Jun Yang, Yongsheng $\mathrm{Xu}$, and seminar participants at the Fields Institute for Mathematical Sciences (Toronto, Canada), ESSEC (Paris, France), the University of Paris-Dauphine (Paris, France), the Georgia Institute of Technology (Atlanta, USA), and Georgia State University (Atlanta, USA) for valuable comments and suggestions. The usual disclaimers apply.

${ }^{\dagger}$ Department of Industrial Engineering and Management, Jerusalem College of Technology, Jerusalem, Israel

${ }^{\ddagger}$ School of Industrial and Systems Engineering, Georgia Institute of Technology, Atlanta, GA 30332

${ }^{\S}$ Department of Risk Management and Insurance, J. Mack Robinson College of Business, Georgia State University, Atlanta, GA 30303
} 


\section{Introduction}

A growing literature in behavioral economics argues that individual behavior often departs from the neoclassical paradigm of complete rationality. A number of studies demonstrate that agents' decisions are significantly influenced by dispositional characteristics such as optimism (see Baker et al, 2005). In this study, we develop a dynamic, structural model of venture capital investment to derive quantitative assessments of the impact of entrepreneurial optimism on the characteristics of venture capital relationships - the economic value they generate, the structures of dynamic contracts between venture capitalists (VCs) and entrepreneurs (ENs), the durations of VC relationships, the manner in which $\mathrm{VC}$ investment is staged over time, and the extent to which EN optimism could mitigate agency costs of risk-sharing between VCs and ENs.

Although the model we develop is potentially applicable in other economic settings, we focus on VC-EN relationships for two principal reasons. First, venture capital is one of the most important mediums through which technological innovation, a key driver of economic growth, is financed. Second, because innovation is often characterized by high levels of uncertainty and differing beliefs about project quality, it is anecdotally suggested that optimism significantly affects VC-EN relationships. If optimism, indeed, influences VC relationships and thus the financing of innovation, it clearly has important aggregate welfare consequences.

In our dynamic principal-agent model, VCs and ENs could have asymmetric beliefs about the intrinsic quality of projects in addition to having asymmetric attitudes towards their risk. We estimate the model parameters by matching a large set of disparate statistics on the distributions of investments, payoffs, risks and returns of VC projects predicted by the model to their observed values in the data. In particular, we estimate the degree of EN optimism or the optimism premium to be $24.6 \%$, which illustrates that the data are consistent with a significant level of EN optimism. The estimated optimism premium also explains the discrepancy between the discount rates used by VCs to value projects $(\sim 40 \%)$, which adjust for optimistic payoff projections by ENs, and the average expected return of $\mathrm{VC}$ projects $(\sim 15 \%)$. EN optimism mitigates the agency costs of risksharing between VCs and EN's by over 20\%, is a key determinant of the durations and economic values of VC relationships, and could explain features of the dynamic contracts between VCs and ENs observed in reality. We derive new, empirically testable implications for the effects of projects' risks, the degrees of uncertainty about their quality, their physical and human capital intensities on the returns, durations, and levels of investment of VC projects as well as the characteristics of contracts between VCs and ENs. The tractability of the model and its ability to match disparate statistics associated with VC projects suggest that it could be a useful tool to value risky ventures. 
In our continuous-time framework a cash-constrained, risk-averse EN with a project approaches a risk-neutral VC for financing. The VC is the manager of a diversified venture capital fund whose capital is competitively supplied by outside investors who care about the fund's expected return on assets in excess of a benchmark in each period. The $\mathrm{VC}$ or the EN could terminate the relationship at any date. All payoffs occur upon termination of the project. ${ }^{1}$ The model's state variable is the project's termination payoff at each date. It represents the total payoff if the project is terminated at that date and is contractible. Both the $\mathrm{VC}$ and the EN possess project-specific skills and neither can commit to supplying them to a third party. ${ }^{2}$

The termination payoff evolves as a log-normal process. The expected growth rate of the termination payoff process has two components: a fixed component that represents the project's intrinsic quality and a discretionary component that is determined by the VC's investments and the EN's efforts over time. The VC and EN have imperfect information about the project's intrinsic quality. The degree of EN optimism is the difference between the EN's and VC's mean assessments of project quality. The VC's prior on the project's quality coincides with the actual distribution of project qualities. The EN is, however, optimistic in that his mean assessment of the project's quality exceeds the actual mean. The VC's and EN's beliefs are common knowledge; that is, the VC and EN "agree to disagree".

The volatility of the termination payoff process is the project's intrinsic risk, which remains invariant through time. Intermediate observations of the project's termination payoff serve as noisy signals of the project's quality, which enable the VC and the EN to update their assessments in a Bayesian manner. The common variance of their mean assessments is the project's transient risk, which is resolved over time.

The EN is provided with inter-temporal incentives to exert effort through a contract that specifies his share of the termination payoff of the project. Assuming the VC has the bargaining power, we derive the equilibrium dynamic contract between the $\mathrm{VC}$ and the EN. The contract describes the VC's investments over time, the EN's path-dependent payoff upon termination, and the inter-temporal performance targets that must be met for the relationship to continue.

Under the equilibrium contract, the change in the EN's promised payoff over any period is an affine function of the change in the termination payoff or "performance" over the period. Conditional on continuation, the VC's proportional investment rate, the sensitivities of the EN's com-

\footnotetext{
${ }^{1}$ Our analysis could be generalized to incorporate intermediate cash flows that are proportional to the termination payoffs without altering our main results.

${ }^{2}$ As in studies such as Kiyotaki and Moore (1997) and Neher (1999), the project's termination payoff at any date is, therefore, less than or equal to its value under hypothetical full commitment that rationally incorporates future physical and human capital investments of the $\mathrm{VC}$ and $\mathrm{EN}$ - the two values only coincide at the equilibrium termination time of the project.
} 
pensation to performance over each period (the pay-performance sensitivities), and the EN's effort in each period are all deterministic functions of time. The component of the change in the EN's promised payoff that does not depend on performance over the period is, however, stochastic and depends on the VC's and EN's current mean assessments of project quality. The optimal contract between the VC and EN predicted by the model shares many features with observed contractual structures: the VC's payoff structure has "debt" and "equity" components, the EN's stake in the project vests over time, and the VC's claim on the project's payoffs allows her to recover her cumulative investments with high probability (Sahlman, 1990, Kaplan and Stromberg, 2003).

We analytically derive properties of the equilibrium contract. The contract balances the costs of risk-sharing between the $\mathrm{VC}$ and the $\mathrm{EN}$ with the positive rents that the $\mathrm{VC}$ is able to extract due to the EN's optimism. Because the degree of EN optimism declines over time as the VC and EN learn about the project's intrinsic quality, the rents from the EN's optimism decrease relative to the costs of risk-sharing. The VC's proportional investment rates, the EN's pay-performance sensitivities, and the EN's effort, therefore, decrease monotonically over time. In the benchmark scenario in which the VC and EN have symmetric beliefs, however, the EN's pay-performance sensitivity and the VC's proportional investment rate are constant over time. Hence, the presence of EN optimism is a key determinant of the inter-temporal variations in the EN's pay-performance sensitivity and the VC's investment rate. The paths of the VC's proportional investment rate, the EN's payperformance sensitivity, and the EN's effort (conditional on continuation of the relationship) all decline with the project's intrinsic and transient risk and increase with the degree of EN optimism. The duration of the relationship increases with the degree of EN optimism and decreases with the EN's risk aversion. These results illustrate the negative effects of risk and the positive effects of EN optimism on risk sharing between the $\mathrm{VC}$ and $\mathrm{EN}$ and, therefore, the power of incentives for the EN. The negative relation between duration and the degree of EN optimism is consistent with the evidence in Kaplan and Stromberg (2003) that experienced entrepreneurs, who are likely to have more realistic beliefs, receive fewer rounds of financing.

Using the simulated method of moments, we estimate the baseline values of the key structural parameters of the model, which include the average intrinsic quality of VC projects, the degree of EN optimism, the EN's cost of bearing risk and his disutility of effort. We estimate these parameters by matching the predicted values of a disparate set of statistics pertaining to the distributions of total investments, payoffs, risks and returns of $\mathrm{VC}$ projects to their observed values reported in Sahlman (1990) and Cochrane (2005). With significantly fewer parameters, the model is able to closely match these observed statistics. Further, the parameter confidence intervals obtained using bootstrapping reveal that the structural parameters of the model are estimated quite accurately. 
Our indirect inference approach shows that the average initial degree of EN optimism implied by the data is $24.6 \%$, which is almost four times the average intrinsic quality, $6.6 \%$, of all projects. We obtain quantitative guidance on the relative impact of EN optimism by also numerically analyzing the "no agency" benchmark scenario in which there are no conflicts of interest between the VC and EN as well as the "symmetric beliefs" benchmark scenario in which the VC and EN have asymmetric attitudes towards risk, but have symmetric beliefs about project quality. We find that, on average, EN optimism mitigates the agency costs of risk sharing by over 20\%. Due to the substantial rents the VC extracts by "feeding" EN optimism, we also find that the benefits of EN optimism more than completely offset the loss in the value of the VC fund's stake in the project due to the agency costs of risk-sharing. The positive effects of EN optimism on project value are consistent with the empirical evidence reported in Gelderen et al (2005).

We numerically establish testable implications of the model by examining how changes to the degree of EN optimism, the project's human capital intensity, and the project's intrinsic and transient risks affect three fundamental characteristics of VC-EN relationships: expected duration, firm value, and net present value of the payoffs to the $\mathrm{VC}$ fund or the VC fund stake. Consistent with our earlier analytical results, EN optimism significantly increases these characteristics. Because the EN's effort is a key driver of the economic value generated by the VC-EN relationship, each of these characteristics also increases with the project's human capital intensity. The project's intrinsic and transient risks have opposing effects on the "speed of learning" about project quality and, therefore, the rate at which the degree of EN optimism declines over time. As a result, they have dramatically opposite effects: each of the three characteristics increases with the project's initial transient risk, but decreases with its intrinsic risk. Hence, firm value and the VC fund's stake are enhanced when there is greater uncertainty about project quality. Consistent with empirical evidence, therefore, VCs have significant incentives to finance innovative projects compared with mature or "imitating" projects because there is greater uncertainty about the quality of innovative projects (Hellman and Puri, 2000). We also find that firm value is positively related to the duration of the relationship, which is consistent with the evidence in Gompers (1995).

It is well-documented in the empirical and anecdotal literature that VCs use discount rates around $40 \%$ to value projects. It has been suggested that higher discount rates could be a mechanism that VCs use to adjust optimistic projections by ENs. Previous research, however, has not ascertained whether EN optimism is, in fact, significant enough to generate such a large discrepancy between the discount rates used by VCs and the average expected return of VC projects, which is approximately 15\% (Cochrane, 2005). We use our structural model to address this issue. We define the implied discount rate as the rate at which the EN's projections of the project's payoffs would 
have to be discounted so that the resulting project value equals the project value under the VC's projections of the project's payoffs. Consistent with the average expected return of VC projects, we assume a discount rate of $15 \%$ to obtain the project value under the VC's projections. The implied discount rates for a wide range of parameter values predicted by the model lie between $40 \%$ and $50 \%$, which is consistent with the range of $\mathrm{VC}$ discount rates reported in prior empirical research (for example, Sahlman, 1990). Our study, therefore, confirms that entrepreneurial "optimism premia" are high enough to justify the discount rates used by VCs in reality. The internal rates of return of $\mathrm{VC}$ projects $(\sim 60 \%)$ as well as the ratio of project values under EN beliefs to those under VC beliefs $(\sim 2)$ predicted by the model also match their corresponding values reported in anecdotal literature (Gladstone and Gladstone, 2002, p. 168).

While the effects of agency conflicts and imperfect information are studied in several contexts by previous studies, theoretical literature that incorporates asymmetric beliefs in the context of the financing of innovation is relatively nascent. Landier and Thesmar (2005) develop a two-period VC model with asymmetric beliefs and show that optimistic entrepreneurs tend to rely on short-term debt rather than long-term debt. Our framework differs significantly from theirs in that investment could be staged over time and the dynamic contract between the VC and the EN has both "equity" and "debt" components. In another contemporaneous working paper, Cuny and Talmor (2005) analyze a two-period VC finance model and show that round financing is preferred to milestone staging when the EN is more optimistic than the VC. We contribute to this stream of the literature by adopting a structural approach to examine how the interplay between asymmetric beliefs and agency conflicts affect the manner in which VC investment is staged over time, the dynamic contract between the $\mathrm{VC}$ and the $\mathrm{EN}$, and the duration and economic value of $\mathrm{VC}$ relationships.

A second stream of the literature investigates the importance of staging in the mitigation of VC-EN agency conflicts. In a deterministic model, Neher (1999) shows that staging is essential to overcome the hold-up problem. As in Neher (1999), the manner in which VC investment is staged over time as well as the number of stages are determined endogenously in our framework. As Neher's (1999) model is fully deterministic, however, his framework cannot be used to study the effects of risk, imperfect information, and asymmetric beliefs on the characteristics of $\mathrm{VC}$ relationships. ${ }^{3}$

A third strand of the literature analyzes the features of the optimal contracts that emerge in "double-sided" two-period moral hazard models in which the VC and EN exert effort (Casamatta, 2003, Cornelli and Yosha, 2003, Schmidt, 2003, Repullo and Suarez, 2004, Inderst and Muller, 2004). We too develop a model in which the VC and EN take value-enhancing actions. Similar to

\footnotetext{
${ }^{3}$ Kockesen and Ozerturk (2004) argue that some sort of EN "lock-in" is essential for staged financing to occur. Egli et al (2006) argue that staging can be used to build an EN's credit rating. Berk et al (2004) develop an R\&D model with a single, monolithic agent in which staging is exogenous.
} 
these studies, the optimal contracts predicted by our analysis have "debt" and "equity" features consistent with observed contractual structures. In contrast, however, our analysis focuses on the effects of asymmetric beliefs on the characteristics of VC-EN relationships. Moreover, our dynamic, structural model can be calibrated to data to obtain quantitative assessments of the degree and effects of entrepreneurial optimism.

Our framework shares features of dynamic principal-agent models with Bayesian learning (for example, Gibbons and Murphy, 1992, Bergemann and Hege, 1998, Holmstrom, 1999). We contribute to this literature by developing a framework where the principal and the agent make investments (physical and human capital) over time, have asymmetric beliefs about project quality, and the relationship is terminated endogenously. The distinction between projects' intrinsic and transient risks that we emphasize in our analysis leads to novel implications for the effects of these two components of risk on VC relationships.

Cochrane (2005) uses an empirical model to estimate the risks and returns of VC projects accounting for the selection bias inherent in the measurement of returns. We complement his study by developing a theoretical structural model in which the underlying economic forces that drive VC projects' investments and payoffs are explicitly incorporated, thereby illuminating the sources as well as the effects of VC projects' risks and returns.

In summary, this study's primary contribution to the literature is the development and analysis of a dynamic structural model of venture capital investment. The model is parsimonious, yet realistic enough to be taken to the data to yield quantitative assessments of the effects of the salient aspects of VC projects, namely, risky payoffs, agency conflicts, uncertainty about project quality and asymmetric beliefs. The predictions of our study are broadly consistent with extant empirical evidence on the characteristics of VC-EN relationships and also suggest additional testable implications that could provide guidance for future empirical research. Our study shows that entrepreneurial optimism is an essential determinant of the characteristics of VC relationships. The tractability of our structural model, coupled with the fact that it is able to match disparate empirical data on the distributions of investments, payoffs, risks and returns of projects as well as the discount rates used to value them, suggests that it could be useful as a tool to value risky ventures whose cash flows are affected by asymmetric beliefs and agency conflicts. 


\section{The Model}

We consider a continuous-time framework with time horizon $[0, T]$. Apart from its analytical tractability, a continuous-time formulation ensures the existence of equilibrium in the model. ${ }^{4}$ At date 0, a cash-constrained entrepreneur (EN) with a project approaches a venture capitalist (VC) for financing. The $\mathrm{VC}$ is the manager of a diversified venture capital fund whose capital is competitively provided by outside investors. We focus on a representative project in the VC fund's portfolio. The project can generate value through physical capital investments by the VC and human capital (effort) investments by the EN. The VC and the EN have imperfect information about the project and differ in their initial assessments of the project's quality.

If the VC agrees to invest in the project, she invests an initial amount of seed capital $V_{0}$. She offers the EN a long-term contract that describes her subsequent investments in the project over time and the EN's compensation. The VC's investments are made continuously over time. The long-term contract between the VC and EN is characterized by "one-sided commitment" in that the $\mathrm{VC}$ is committed to the contract, but the EN could renege on the contract at any date.

The key state variable in the model is the project's termination payoff $V_{t}$, which is the total payoff if the VC-EN relationship is terminated at date $t$. The termination payoff is the value of the project "outside the VC-EN relationship". Similar to Neher (1999), the VC and the EN possess project-specific skills that are not transferrable. Thus, the termination payoff (the "outside" value) of the project is, in general, less than its "inside" value (see also Kiyotaki and Moore, 1997). The termination payoff is the only economic variable that is contractible. ${ }^{5}$ For simplicity, we assume the project does not generate any intermediate cash flows. All our results hold in an extension of the model in which the project generates intermediate cash flows that are proportional to the termination payoff process.

\subsection{The Termination Payoff Process}

The initial termination payoff of the project equals the initial seed capital investment $V_{0}$ of the VC. The incremental termination payoff, that is, the change in termination payoff over any period $[t, t+d t], d V_{t}$, depends on the physical capital investment of the $\mathrm{VC}$, the human capital (effort) investment of the EN, the intrinsic quality of the project, and its risk and is given by

$$
d V_{t}=\overbrace{\Phi\left(c_{t}, \eta_{t}\right) V_{t} d t}^{\text {discretionary output }}-\overbrace{l_{t} V_{t} d t}^{\text {operating costs }}+\overbrace{\Theta V_{t} d t}^{\text {intrinsic output }}+\overbrace{s V_{t} d B_{t}}^{\text {intrinsic risk }} .
$$

\footnotetext{
${ }^{4}$ As discussed by Holmstrom and Milgrom (1987), optimal contracts need not exist in discrete-time frameworks in which payoffs are drawn from distributions with unbounded support.

${ }^{5}$ An important stream of the literature examines the effect of "incomplete contracting" on VC relationships (Kirilenko, 2001, Dessein, 2005).
} 
In (1), $B$ is a standard Brownian motion, and $c_{t} V_{t} d t$ and $\eta_{t} V_{t} d t$ represent, respectively, the capital invested by the VC and the EN's effort exerted in period $[t, t+d t]$. Since $c_{t}$ and $\eta_{t}$ are the investment and effort rates as proportions of the state variable $V_{t}$, we shall hereafter refer to $c_{t}$ and $\eta_{t}$ as simply the investment rate and effort, respectively, to simplify the exposition. If either the EN's effort or the VC's investment is zero, then the change in termination payoff is given by $d V_{t}=-l_{t} V_{t} d t$. It is not difficult to show that, in equilibrium, effort and investment are both positive if the project continues. To simplify the notation, we henceforth assume the termination payoff evolves as in (1).

The modeling of the termination payoff as a log-normal (rather than normal) process is motivated by the observations in Cochrane (2005) that venture capital payoffs are highly skewed so that modeling them as normal processes would be inappropriate. We now discuss the four sources of the change in the termination payoff in each period.

Discretionary output: Discretionary output in period $[t, t+d t]$ is a direct result of the VC's investment rate $c_{t}$ and the EN's effort $\eta_{t}$, and is determined by the production function $\Phi\left(c_{t}, \eta_{t}\right)=A c_{t}^{\alpha} \eta_{t}^{\beta}$. The VC closely monitors the EN so that the EN's effort is observable to the VC. ${ }^{6}$ However, it is non-verifiable by a third party and, therefore, not directly contractible. Hence, the EN must be provided with appropriate incentives to exert effort through her explicit contract with the VC.

Operating costs: The term $l_{t} V_{t} d t$ represents operating costs, which could include depreciation expenses, decline in revenues due to increased competition, fixed costs arising from increases in the scale of the project, etcetera. The operating costs parameter $l_{t}$ is deterministic, increasing and convex over time, which ensures that termination occurs in finite time almost surely.

Intrinsic risk: The term $s V_{t} d B_{t}$, where $s>0$ is constant, represents the "intrinsic" component of the project's risk in period $[t, t+d t]$. The intrinsic risk $s$ is the component of the project's risk that remains invariant over time.

Intrinsic output: The parameter $\Theta$ represents the growth rate of the project's termination payoff arising from the project's intrinsic quality. The VC and the EN have imperfect information about $\Theta$ and could also differ in their beliefs about its value. As in a large number of studies that incorporate heterogeneous beliefs (for example, Allen and Gale, 1999), their respective beliefs are common knowledge; that is, they "agree to disagree". The uncertainty in the value of $\Theta$ may be viewed as the project's transient risk. The transient risk is resolved over time as the VC and the EN update their priors on $\Theta$ in a Bayesian manner based on observations of the project's

\footnotetext{
${ }^{6}$ Sahlman (1990) reports that venture capitalists closely monitor the firms they invest in. He mentions that lead venture investors visit each company in their portfolio 19 times every year and spend 100 hours in direct contact. Hellman and Puri (2000, 2002) emphasize the importance of monitoring and oversight in their empirical analyses of venture capital financing.
} 
performance. The VC's and EN's initial priors on $\Theta$ are normally distributed with $\Theta \sim N\left(\mu_{0}^{V C}, \sigma_{0}^{2}\right)$ and $\Theta \sim N\left(\mu_{0}^{E N}, \sigma_{0}^{2}\right)$, respectively. The VC's beliefs are correct so that $\Theta$ is actually drawn from the distribution $N\left(\mu_{0}^{V C}, \sigma_{0}^{2}\right)$. Define

$$
\xi_{t} d t:=d \ln V_{t}-\left(\Phi\left(c_{t}, \eta_{t}\right)-0.5 s^{2}-l_{t}\right) d t=\Theta d t+s d B_{t}
$$

Since the VC's investment rate $c_{t}$ and the EN's effort $\eta_{t}$ are observable, it follows from well-known formulae (Oksendal 2003) that the posterior distribution on $\Theta$ for each date $t \geq 0$ is $N\left(\mu_{t}^{\ell}, \sigma_{t}^{2}\right)$, $\ell=V C, E N$, where

$$
\begin{aligned}
\sigma_{t}^{2} & =\frac{s^{2} \sigma_{0}^{2}}{s^{2}+t \sigma_{0}^{2}} \\
\mu_{t}^{\ell} & =\frac{s^{2} \mu_{0}^{\ell}+\sigma_{0}^{2} \int_{u=0}^{t} \xi_{u} d u}{s^{2}+t \sigma_{0}^{2}}, \ell=V C, E N .
\end{aligned}
$$

From Oksendal (2003), we can show that the evolution of the mean posterior assessment of project quality, $\mu_{t}^{\ell}$, is described by the following stochastic differential equation

$$
d \mu_{t}^{\ell}=\frac{\sigma_{0}^{2}}{s^{2}+t \sigma_{0}^{2}}\left[d \ln V_{t}-\left(\Phi\left(c_{t}, \eta_{t}\right)-0.5 s^{2}-l_{t}\right) d t-\mu_{t}^{\ell} d t\right], \ell=V C, E N .
$$

From (1) and (5), the standard deviation $\sigma_{t}^{\mu}$ of the evolution of the mean assessment of project quality is

$$
\sigma_{t}^{\mu}=\frac{s \sigma_{0}^{2}}{s^{2}+t \sigma_{0}^{2}}
$$

Note that the standard deviations of the evolutions of the VC's and EN's mean assessments of project quality are equal and decrease with time. Let

$$
\Delta_{t}:=\mu_{t}^{E N}-\mu_{t}^{V C}=\frac{s^{2} \Delta_{0}}{s^{2}+t \sigma_{0}^{2}}=\frac{\sigma_{t}^{2}}{\sigma_{0}^{2}} \Delta_{0}
$$

denote the degree of asymmetry in beliefs at date $t$. It follows from (7) that the degree of asymmetry in beliefs is resolved deterministically over time, and there is a linear relationship between the resolution of the asymmetry of beliefs and the resolution of the transient risk. Consistent with the evidence reported by Landier and Thesmar (2005) and Sahlman (1990), the EN is initially optimistic so that $\Delta_{0} \geq 0$. Thus, $\Delta_{t}$ decreases with time since the variance $\sigma_{t}^{2}$ decreases with time.

\subsection{Contracting between the VC and EN}

We denote the information contained in the history of termination payoffs $\left\{V_{t}, t \geq 0\right\}$ by $\left\{\mathcal{F}_{t}\right\}$. Since the project does not generate intermediate cash flows, the contract between the VC and the EN describes the VC's incremental capital investments over time and the payoffs to be received by both parties upon termination. Further, since the EN could choose to terminate the relationship 
at any date, the contract specifies the payoffs to be received by both parties if the project were terminated at any date $t \geq 0$. More precisely, a feasible contract is described by the triplet $(P, c, \tau)$, where $P$ and $c$ are $\left\{\mathcal{F}_{t}\right\}$-adapted stochastic processes and $\tau$ is an $\left\{\mathcal{F}_{t}\right\}$-stopping time. $P_{t}$ is the EN's payoff and $V_{t}-P_{t}$ is the VC fund's payoff if the relationship is terminated at date $t \geq 0, c_{t}$ is the VC's investment rate at date $t$, and $\tau$ is the termination time. The termination time is stochastic and $V_{\tau}-P_{\tau}$ is the payoff to the VC fund upon termination, and not the VC's compensation.

The VC possesses the bargaining power with the EN at date zero and offers the EN a long-term contract, which specifies her dynamic capital investments in the projects, the termination time, and the EN's payoff upon termination. The EN, in turn, dynamically chooses his effort to maximize her total expected utility including her disutility from effort. ${ }^{7}$ The fact that the EN could repudiate the contract at any date allows him to extract a portion of the surplus generated by the relationship over time. The equilibrium contract is self-enforcing on the EN, that is, the EN has no incentive to renege prior to the termination time specified in the contract.

\subsection{The VC's Compensation Structure and Objective}

The VC is the manager of a venture capital fund with capital supplied by outside investors. ${ }^{8}$ The VC could set up and manage multiple diversified funds over time. Outside investors (existing and potential) in the market evaluate the $\mathrm{VC}$ and provide capital to her on the basis of the expected return she earns on assets in excess of a benchmark return, that is, her risk-adjusted return on assets. As we focus on a representative project in the VC's portfolio, we define the VC's relative performance in terms of the performance of the representative project.

As discussed earlier, the project's outside value or asset value is $V_{t}$ at any date $t$. The value of the fund's holdings in the project at date $t$ is the termination payoff less the promised payoff to the EN, i.e., $V_{t}-P_{t}$. After subtracting the capital investment $c_{t} V_{t} d t$ specified by the contract, the change in the value of the fund's holdings over the period $[t, t+d t]$ (if the project is continued) is

$$
\text { Change in Value of Holdings }=\left[V_{t+d t}-V_{t}\right]-\left[P_{t+d t}-P_{t}\right]-c_{t} V_{t} d t \text {. }
$$

Suppose, instead, that the project's assets were (hypothetically) invested in the benchmark. The change in the value of the fund's holdings would be given by

Change in Value of Holdings under Benchmark $=\left[V_{t}\left(1+R_{b} d t\right)-V_{t}\right]-\left[P_{t}(1+r d t)-P_{t}\right]$.

\footnotetext{
${ }^{7}$ As is common in the contract theory literature, we could alter the notation to allow for the EN's dynamic effort choices to also be specified by the contract, which satisfies incentive constraints for the EN.

${ }^{8}$ We could view the VC as the representative "general partner" of the fund and the outside investors as the "limited partners". Gompers and Lerner (1996) document that, starting from the early 1980s, a majority of VC organizations were formed as limited partnerships so that, by the year 1992, funds formed $80 \%$ of the total VC pool.
} 
The term $V_{t}\left(1+R_{b} d t\right)$ is the end-of-period value of the project's assets if they are invested in the benchmark. The term $P_{t}(1+r d t)$ is the end-of-period promised payoff to the EN, which is his beginning-of-period promised payoff $P_{t}$ carried forward at the risk-free rate $r$, an assumed constant.

The change in value of the fund's holdings in excess of the benchmark over the period is the difference between the right-hand sides of (8) and (9), respectively. The Excess Return On Assets (ExROA) of the fund over the period is the ratio of the change in value of the fund's holdings in excess of the benchmark to the asset value $V_{t}$, and is given by

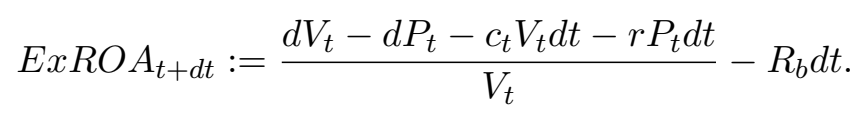

Note that this excess return on assets is gross of the fund's operating costs and the VC's compensation over the period. The VC's payoff in each period is an increasing affine function of the expected excess return on assets, and is given by

$$
V C \text { 's Payoff } t_{t+d t}:=A+B E_{t}^{V C}\left(\operatorname{ExROA}_{t+d t}\right), B>0 .
$$

Outside investors have the same beliefs as the VC so that the expectation is under the VC's beliefs. ${ }^{9}$

In Appendix A, we present an extension of the model in which the dynamic relationship between outside investors and the $\mathrm{VC}$ is incorporated. Outside investors competitively supply capital to the $\mathrm{VC}$ and the $\mathrm{VC}$ faces decreasing returns to scale in managing her investments. In equilibrium, the expected excess return on assets net of operating costs and the VC's compensation is zero, that is, outside investors earn competitive or zero risk-adjusted excess returns on their investments. We show that the (endogenously derived) capital the $\mathrm{VC}$ is able to raise at date $t$ is an increasing function of the expected excess return on assets. Effectively, the expected excess returns on assets generated by the VC over time serve as signals of her investment ability for which she is dynamically compensated by outside investors through the capital they provide.

For concreteness and simplicity, and to facilitate the structural estimation of the model in Section 7, we assume the VC's operating costs are quadratic in the total capital she manages and that she receives a constant proportion of the capital she manages as compensation in each period. It then follows that the VC's compensation has the affine form (11) (see Appendix A). ${ }^{10}$ Since the fixed portion of the VC's compensation neither influences nor is affected by her actions, we hereafter

\footnotetext{
${ }^{9}$ The VC is allowed to have unlimited liability. Evidence reported by Sahlman (1990) shows that, unlike the limited partners, general partners of VC funds have unlimited liability.

${ }^{10}$ Our analytical results hold more generally if the VC's compensation is any increasing, deterministic function of the assets under management. In reality, VCs receive a proportion of the fund's committed capital and a percentage of the fund's profits over its lifetime (Sahlman, 1990) so that the VC's compensation increases with measures of the fund's performance. Because our focus in this paper is on the VC-EN relationship, we simplify the analysis by assuming a simple "constant proportion of assets" payoff structure as in the case of mutual fund managers. The VC's compensation, therefore, increases with her performance as represented by the capital she manages.
} 
set $A=0$ to simplify the notation. Further, because $B$ is merely a constant of proportionality, we henceforth set it equal to one.

The VC is risk-neutral with respect to her payoffs that are proportional to the expected excess returns on assets. Hence, her discounted expected utility from the project until termination is

$$
\begin{aligned}
\text { VC's Expected Utility } & =E_{0}^{V C}\left[\int_{0}^{\tau} e^{-r t} E_{t}^{V C}\left(E x R O A_{t+d t}\right)\right] \\
& =E_{0}^{V C}\left[\int_{0}^{\tau} e^{-r t} E x R O A_{t+d t}\right] \\
& =E_{0}^{V C}\left[\int_{0}^{\tau} e^{-r t}\left(\frac{d V_{t}-d P_{t}-c_{t} V_{t} d t-r P_{t} d t}{V_{t}}-R_{b} d t\right)\right],
\end{aligned}
$$

where the expectation is under the VC's beliefs about the project's intrinsic quality. The second equality above follows from the law of iterated expectations and the third follows from (10).

As will become clear shortly, the forms of the VC's objective function described by (12), as well as the EN's objective described in the next subsection, contribute significantly to the tractability of the model. In particular, the fact that the $\mathrm{VC}$ maximizes a discounted stream of payoffs that depend on the expected excess return on assets eliminates complex path-dependencies in the equilibrium arising from the fact that the termination payoff process is log-normal and, therefore, persistent. In addition to the fact that it corresponds with real-life venture capital partnerships, the modeling of the VC as the manager of a venture capital fund with capital supplied by outside investors provides an internally consistent theoretical foundation for the VC's objective function (12).

\subsection{The EN's Objective}

As in several studies in the principal-agent literature, the EN has linear inter-temporal preferences with a subjective discount rate (for example, DeMarzo and Fishman, 2004). In contrast with these studies, however, the EN's subjective discount rate is stochastic, which directly reflects his costs of bearing risk (see Alvarez and Jermann, 2000). The EN incurs an additive monetary disutility of effort. More precisely, if the EN's termination payoff is $P_{\tau}$ and his effort is described by the process $\left\{\eta_{t}\right\}$, his subjective valuation of his future payoffs (including his disutility of effort) at date zero is

$$
U_{0}=E_{0}^{E N}\left[e^{-r \tau-\frac{1}{2} \lambda^{2} \tau-\lambda B_{\tau}} P_{\tau}-\int_{0}^{\tau} e^{-r t-\frac{1}{2} \lambda^{2} t-\lambda B_{t}} k \eta_{t}^{\gamma} V_{t} d t\right]
$$

In (13), $e^{-r t-\frac{1}{2} \lambda^{2} t-\lambda B_{t}}$ is the stochastic discount factor by which the EN discounts a payoff at date $t$, where $\lambda>0$ measures his cost of bearing risk. A nonzero cost of risk $\lambda$ in the EN's subjective valuation (13) of his future payoffs (or his stake in the firm) reflects his imperfect access to outside credit markets. ${ }^{11}$ In Section 6, we estimate $\lambda$ by matching the model's predictions to data. The

\footnotetext{
${ }^{11}$ In analogy with fundamental results in asset pricing theory (Duffie, 2001), we directly model the EN's subjective
} 
expectation in (13) is with respect to the EN's beliefs about the project's intrinsic quality. The term $k \eta_{t}^{\gamma} V_{t} d t ; k>0$ represents the EN's disutility of effort in period $[t, t+d t]$. The EN's disutility of effort increases with the scale of the project represented by its termination payoff. This is consistent with the fact that the expected change in termination payoff in (1) due to investment and effort is also proportional to the termination payoff. As in the case of the termination payoff, the EN values his cost of effort in any period by its expectation weighted by the stochastic discount factor. In Appendix A, we show that the EN's preferences as described by (13) actually belong to the general class of recursive or "stochastic differential" preferences (Duffie and Epstein, 1992).

In our analysis, we find it useful to express the EN's valuation (13) of his future payoffs in an alternate form. The process $\exp \left(-\frac{1}{2} \lambda^{2} t-\lambda B_{t}\right)$ is a square-integrable martingale, and is the Radon-Nikodym derivative process of a new probability measure equivalent to the original one (see Chapter 6 of Duffie, 2001). The EN's valuation (13) is then expressed as

$$
U_{0}=\bar{E}_{0}^{E N}\left[e^{-r \tau} P_{\tau}-\int_{0}^{\tau} e^{-r t} k \eta_{t}^{\gamma} V_{t} d t\right]
$$

where the expectation above is under the new probability measure. From (14), we can express the EN's objective by assuming that he is risk-neutral under a risk-adjusted probability - the EN's valuation probability and not the actual probability - that reflects his costs of bearing risk.

For future reference we note that by Girsanov's theorem (see Chapter 6 of Duffie, 2001), the termination payoff process evolves under the EN's valuation probability as

$$
d V_{t}^{E N}=\left(\Phi\left(c_{t}, \eta_{t}\right)-l_{t}-\lambda s\right) V_{t} d t+\Theta V_{t} d t+s V_{t} d B_{t}^{E N}
$$

where

$$
B_{t}^{E N}=B_{t}+\lambda t
$$

is a Brownian motion under the EN's valuation probability. The superscripts on this process and the termination payoff process indicate that the evolution (15) is in the EN's valuation probability.

\subsection{The VC-EN Interaction}

At each date $t$, the EN could terminate the relationship with the VC and receive his promised payoff $P_{t}$. The EN chooses to continue the relationship over the next infinitesimal time period if and only if his expected utility from continuation exceeds his utility from termination. The continuation value of the $\mathrm{EN}$ at date $t, C U_{t}$, is

$$
C U_{t}:=\bar{E}_{t}^{E N}\left[e^{-r(\tau-t)} P_{\tau}-P_{t}-\int_{t}^{\tau} e^{-r(u-t)} k \eta_{u}^{\gamma} V_{u} d u\right],
$$

valuation of his stake in the firm as the expectation of his future total payoffs weighted by a subjective stochastic discount factor or "valuation kernel". 
where $\bar{E}_{t}^{E N}$ denotes the EN's expectation under his valuation probability conditioned on the information available at date $t$.

From (12), the risk-neutral VC's continuation value $C V_{t}$ at date $t$ is given by

$$
C V_{t}:=E_{t}^{V C}\left[\int_{t}^{\tau} e^{-r(u-t)}\left(\frac{d V_{u}-d P_{u}-c_{u} V_{u} d u-r P_{u} d u}{V_{u}}-R_{b} d u\right)\right],
$$

where $E_{t}^{V C}$ denotes the expectation under the VC's beliefs conditioned on the information available at date $t$. The equilibrium termination (stopping) time is the first time at which her continuation value hits zero.

\section{Equilibrium}

We assume the following conditions on the parameters:

Assumption $1 \quad(1-\alpha) \gamma / \beta>2$.

Assumption $2 \quad \Delta_{0}<\lambda s$.

These conditions, which are easily met in the calibrated model, ensure that an equilibrium contract between the VC and the EN exists. Assumption 1 ensures that the curvature of the EN's disutility of effort is above a threshold relative to the sensitivity of output to his effort. Assumption 2 ensures that EN optimism is not high enough to outweigh the costs of risk-sharing.

\subsection{Existence and Characterization of Equilibrium}

In Appendix B (see Lemma 1), we show that the optimal contract has the following affine form:

$$
d P_{t}=a_{t} V_{t} d t+b_{t} d V_{t}
$$

In (19), the contractual parameters $a_{t}, b_{t}>0$ are $\left\{\mathcal{F}_{t}\right\}$-measurable. It follows easily from (19) that the EN's contract $P$ satisfies

$$
P_{\tau}=P_{0}+\int_{0}^{\tau}\left[a_{t} V_{t} d t+b_{t} d V_{t}\right]
$$

In Lemma 1 in Appendix B, we also show that the optimal long-term contract between the VC and the EN can be implemented by a sequence of single-period contracts, which are negotiated at each date $t$ with the VC making "take it or leave it" offers to the EN (see Fudenberg et al, 1990). We can, therefore, use backward induction to characterize the equilibrium. We now provide a heuristic derivation of the equilibrium. To simplify the exposition we deliberately sacrifice some mathematical rigor. In Appendix B, we provide the detailed mathematical arguments underlying the derivation of the equilibrium. 
Assume for the purpose of this heuristic discussion that the time interval $d t$ between successive dates is discrete rather than infinitesimal. Suppose that the project has not been terminated as of the date $T-d t$. Recall that the EN and VC priors on $\Theta$ as of date $T-d t$ are $N\left(\mu_{T-d t}^{\ell}, \sigma_{T-d t}^{2}\right)$ with $\mu_{T-d t}^{\ell}$ and $\sigma_{T-d t}^{2}$ given by (4) and (3), respectively with the index $t$ set to $T-d t$. Keep in mind that the VC's and EN's initial beliefs and, therefore, their respective beliefs at any date are common knowledge. For subsequent convenience in our inductive derivation of the equilibrium, it will be convenient to use the index $t$ to denote the date. The index $t=T-d t$ for now, but it will later denote an arbitrary date when we establish the inductive step in our analysis.

\section{The EN's Optimal Effort in Period $[T-d t, T]$ for a Given Contract}

Suppose that in period $[t, t+d t]$ (recall that $t=T-d t$ ), the VC's investment rate is $c$ and the EN's contractual parameters are $(a, b)$ in (19). If the EN's effort is $\eta$ in period $[t, t+d t]$, his continuation value (17) is given by

$$
C U_{t}=\bar{E}_{t}^{E N}\left[e^{-r d t}\left(P_{t}+d P_{t}\right)-P_{t}-k \eta^{\gamma} V_{t} d t\right]=\bar{E}_{t}^{E N}\left(a V_{t} d t+b d V_{t}-k \eta^{\gamma} V_{t} d t-r P_{t} d t\right),
$$

where the second equality follows from (19) (neglecting $o(d t)$ terms). From (15), we have

$$
C U_{t}=\left[\left(a+b\left(A c^{\alpha} \eta^{\beta}-l_{t}+\mu_{t}^{E N}-\lambda s\right)-k \eta^{\gamma}\right) V_{t}-r P_{t}\right] d t
$$

By Assumption 1, the optimal effort level exists and is given by

$$
\eta(b, c):=\left(\frac{A \beta c^{\alpha} b}{\gamma k}\right)^{\frac{1}{\gamma-\beta}} .
$$

\section{The VC's Choice of Contract in Period $[T-d t, T]$}

The VC will choose her investment rate $c$ and the EN's contractual parameters $(a, b)$ that will ensure the EN's participation and also rationally anticipate the EN's best effort response. Since the $\mathrm{VC}$ has the bargaining power and can make a "take it or leave it" offer to the EN, it is optimal for her to choose $(a, b)$ so that regardless of the state at date $t=T-d t$

$$
C U_{t} \equiv 0
$$

It follows from (22) that the relation between the parameters $a, b$, and $c$ in period $[t, t+d t]$ that ensures (24) is given by

$$
a(b, c):=k \eta(b, c)^{\gamma}-b\left(A c^{\alpha} \eta(b, c)^{\beta}-l_{t}+\mu_{t}^{E N}-\lambda s\right)+r \frac{P_{t}}{V_{t}} .
$$

Incorporating the EN's best effort response, the VC's continuation value (18) at date $t$ is

$$
C V_{t}=E_{t}^{V C}\left[\frac{(1-b) d V_{t}-a(b, c) V_{t} d t-c V_{t} d t-r P_{t} d t}{V_{t}}-R_{b} d t\right]
$$


Substituting the EN's optimal effort (23) into (26),

$$
C V_{t}=\Lambda_{t}(b, c) d t
$$

where

$$
\Lambda_{t}(b, c):=\Delta_{t} b-\lambda s b+\phi(b) c^{\alpha \frac{\gamma}{\gamma-\beta}}-c+\mu_{t}^{V C}-l_{t}-R_{b} .
$$

In (28), $\Delta_{t}$ is the degree of asymmetry in beliefs at date $t$, defined in (7), and

$$
\phi(b):=A^{\frac{\gamma}{\gamma-\beta}}\left(\frac{1}{k}\right)^{\frac{\beta}{\gamma-\beta}}\left(\left(\frac{\beta b}{\gamma}\right)^{\frac{\beta}{\gamma-\beta}}\left(1-\frac{\beta b}{\gamma}\right)\right) .
$$

The VC chooses the capital investment rate $c$ and the EN's pay performance sensitivity $b$ to maximize $\Lambda_{t}(b, c)$. We first determine the VC's optimal investment rate $c(b)$ as a function of the EN's pay-performance sensitivity $b$. We then derive the optimal pay-performance sensitivity and the corresponding investment rate.

The function $\phi(b)$ in $(29)$ is nonpositive when $b \geq \gamma / \beta$. Given that $\Delta_{t}$ decreases with $t$, it follows directly from Assumption 2 and (28) that the VC will never choose a pay performance sensitivity $b \geq \gamma / \beta$. For $b \in(0, \gamma / \beta), \phi(b)>0$ and Assumption 1 guarantees that $\Lambda_{t}(b, \cdot)$ is strictly concave in $c$ since the exponent on $c$ is guaranteed to be less than 1 . Consequently,

$$
\begin{aligned}
c(b) & =\hat{K} \phi(b)^{\frac{\gamma-\beta}{(1-\alpha) \gamma-\beta}} \\
\Lambda_{t}(b, c(b)) & =\Delta_{t} b-\lambda s b+K c(b)+\left(\mu_{t}^{V C}-l_{t}-R_{b}\right) .
\end{aligned}
$$

The constants $\hat{K}$ and $K$ in (30) and (31) are positive and depend on $\alpha, \beta, \gamma$ and $A$. We conclude that the $\mathrm{VC}$ will choose the pay performance sensitivity in period $[t, t+d t]$ to solve

$$
b_{t}^{*}:=\arg \max _{0<b<\gamma / \beta} \Lambda_{t}(b, c(b))
$$

In addition, we have the following characterization.

\section{Proposition 1}

(a) Under Assumption 1 the optimal investment function $c(\cdot)$ is positive, increasing and strictly concave on $(0,1]$, is decreasing on $[1, \gamma / \beta]$ and therefore achieves its maximum at $b=1$.

(b) Under Assumptions 1 and 2 there is a unique solution $b_{t}^{*} \in(0,1)$ to $(32)$.

The proof of Proposition 1 and the proofs of all remaining results are in Appendix C. 


\section{The Inductive Step}

We now set $t=T-2 d t$, and suppose the project has not been terminated as of date $(T-2 d t)$. If the VC's investment rate is $c$, the EN's contractual parameters are $(a, b)$, and he exerts effort $\eta$, his continuation value (17) is given by

$$
\begin{aligned}
C U_{t} & =\bar{E}_{t}^{E N}\left[\left(a V_{t} d t+b d V_{t}-k \eta^{\gamma} V_{t} d t-r P_{t} d t\right)+e^{-r d t} C U_{t+d t}\right] \\
& =\bar{E}_{t}^{E N}\left[\left(a V_{t} d t+b d V_{t}-k \eta^{\gamma} V_{t} d t-r P_{t} d t\right)\right]
\end{aligned}
$$

The first line above follows by the law of iterated expectations and the second line follows from (24). Since (33) is identical to (21), our previous arguments show that the EN's optimal effort $\eta(b, c)$ is given by $(23)$ and the component $a(b, c)$ of the EN's compensation is given by $(25)$.

It remains to determine the VC's optimal choices for the investment rate and pay performance sensitivity. Incorporating the EN's best effort response, the VC's continuation value at date $t$ is (see 18)

$$
\begin{aligned}
C V_{t} & =E_{t}^{V C}\left[\frac{(1-b) d V_{t}-a(b, c) V_{t} d t-c V_{t} d t-r P_{t} d t-R_{b} V_{t} d t}{V_{t}}+e^{-r d t} \max \left(C V_{t+d t}, 0\right)\right] \\
& =\Lambda_{t}(b, c) d t+e^{-r d t} E_{t}^{V C} \max \left(C V_{t+d t}, 0\right)
\end{aligned}
$$

where the second line follows from (26) and (27). Since the EN's effort is observable, the updated assessments of project quality at date $t+d t, \mu_{t+d t}^{E N}$ and $\mu_{t+d t}^{V C}$, do not depend on the VC's investment or the EN's effort over the period $t+d t$. It then follows from (27) and (28) that the second term on the right-hand side of (34) does not depend on the EN's effort or the VC's investment at date $t$. Hence, the VC's continuation value at date $t$ is maximized when the optimal investment is given by (30) and the optimal pay performance sensitivity solves (32).

We can clearly extend the above arguments by induction to any date $t$ and thereby derive the equilibrium, as characterized in the following theorem.

\section{Theorem 1 (Characterization of Equilibrium)}

Under Assumption 1, if the project has not been terminated as of date $t \in[0, T]$, then an equilibrium contract offered by the VC and the EN's effort in the period is characterized, as follows:

- The pay performance sensitivity is $b_{t}^{*}$, the solution to (32);

- The investment rate is $c_{t}^{*}:=c\left(b_{t}^{*}\right)$ where $c(\cdot)$ is defined in (30);

- The fixed portion of the EN's compensation is $a_{t}^{*}:=a\left(b_{t}^{*}, c_{t}^{*}\right)$ defined in (25);

- The optimal effort level is $\eta_{t}^{*}:=\eta\left(b_{t}^{*}, c_{t}^{*}\right)$ defined in (23). 
- The VC's maximum continuation value at date $t$ is given by

$$
C V_{t}=\overbrace{\Lambda_{t}\left(b_{t}^{*}, c_{t}^{*}\right) d t}^{\text {within-period flow }}+e^{-r d t} \overbrace{E_{t}^{V C}\left[\max \left\{C V_{t+d t}, 0\right\}\right]}^{\text {future option value }} .
$$

\subsection{The VC's Periodic Flow}

Since the degree of asymmetry in beliefs, $\Delta_{t}$, and variance, $\sigma_{t}^{2}$, are deterministic functions of time (see 7) it follows from Proposition 1 and Theorem 1 that the equilibrium values for the pay performance sensitivity, investment and effort at each point in time (conditional upon continuation) are positive and also deterministic. The only component of the contract that is stochastic and is adjusted based on realizations of the termination payoff $V_{t}$ of the project (the "signal" of project quality) is the component $a_{t}^{*}$ of the EN's compensation. Furthermore, the equilibrium described in Theorem 1 is stable; that is, the EN's pay performance sensitivities, effort, and the VC's investment rates are continuous functions of the model parameters.

As summarized in Theorem 1, the equilibrium contract at date $t$ is determined by $b_{t}^{*}$. Let

$$
F_{t}(b):=\Lambda_{t}(b, c(b))-\mu_{t}^{V C}+l_{t}+R_{b}=\Delta_{t} b-p b+K c(b)
$$

and define

$$
F_{t}^{*}:=\max _{0<b<1} F_{t}(b)
$$

Clearly, the solution to (32) is also a solution to (37). The VC's periodic flow function, $F_{t}(b)$, consists of three components:

Rent from the EN's optimism. The term, $\Delta_{t} b$, reflects the rents that the VC extracts from the EN by exploiting his optimism about the project's intrinsic quality.

Cost of risk. The term, $\lambda s b$, reflects the VC's costs of risk-sharing with the risk-averse EN. We refer to $\lambda s$ as the price of risk.

Return on investment. The "return on investment" term, $K c(b)$, reflects the VC's expected return as a result of her investment and the EN's effort.

\subsection{Comparisons with Observed Contractual Structures}

The equilibrium contract between the VC and the EN has many of the features observed in actual venture capital contractual structures. By (19), the change in the EN's stake in the project over any period $[t, t+d t]$ has a component $a_{t}^{*} V_{t} d t$, which is known at date $t$, and a risky component $b_{t}^{*} d V_{t}$, which is a random variable whose value is realized at date $t+d t$. Because the termination payoff process grows in expectation if the project is continued, and the $\mathrm{VC}$ has the bargaining power, the 
parameter $a_{t}^{*}$ is generally negative. Hence, the component $a_{t}^{*} V_{t} d t$ is similar to a "debt" or payment made by the EN while the component $b_{t}^{*} d V_{t}$ is the "equity" portion of the EN's compensation over the period. Recall, however, that all payoffs occur upon termination so that no payments are actually made by the EN prior to termination. The portion $\int_{0}^{\tau} a_{t}^{*} V_{t} d t$ of the EN's termination payoff could be viewed as a cumulative debt or dividend payment from the EN to the VC fund while the portion $\int_{0}^{\tau} b_{t}^{*} d V_{t}$ is the cumulative outcome of the changes in the EN's equity stake over each period. ${ }^{12}$

Because the VC fund's stake in the project at any date $t$ is $V_{t}-P_{t}$, the VC fund's payoff at termination is $-\int_{0}^{\tau} a_{t}^{*} V_{t} d t+\int_{0}^{\tau}\left(1-b_{t}^{*}\right) d V_{t}$. The $\mathrm{VC}$ fund, therefore, holds a contract that has debt as well as equity components. These features of the optimal contract are consistent with data on observed VC contracts reported by Sahlman (1990) and Kaplan and Stromberg (2003). They document that the most commonly observed security held by VCs is preferred stock in which VC investors hold a claim to a preferred dividend stream (that could be deferred) as well as an equity claim to any residual value of the venture. The complex path-dependence of the VC fund's and EN's payoffs, however, implies that the equilibrium contract between the VC and the EN can only be implemented (or approximated) using combinations of different financial securities, which is also consistent with the evidence in Kaplan and Stromberg (2003).

Because the VC has the bargaining power, we observe that the VC fund's claim to the firm's payoffs ensures that it recovers the cumulative investment $\int_{0}^{\tau} c_{t}^{*} V_{t} d t$ with very high probability. This prediction is also consistent with empirical evidence that the claims of VCs in liquidation are at least as large as their original investments (see Section 3.4 of Kaplan and Stromberg, 2003). The probability that the $\mathrm{VC}$ fund does not recover its cumulative investment is, however, not negligible, especially if the project is terminated early because of poor intermediate realizations of the termination payoff. This is consistent with the evidence in Cochrane (2005) that a nonzero proportion (about nine percent) of VC projects fail to return their investments.

Another salient feature of venture capital agreements is the vesting of the EN's stake in the firm over time. In our framework, if the EN terminates the agreement at some date $s<\tau$, his payoff is $P_{s}=\int_{0}^{s}\left[a_{t}^{*} V_{t} d t+b_{t}^{*} d V_{t}\right]$, which is lower, in expectation, than his payoff $P_{\tau}$ if he were to continue the relationship until the optimal termination time $\tau$.

\footnotetext{
${ }^{12}$ We can generalize the model to allow for intermediate cash flows that are proportional to the termination payoffs. In this generalization, the equilibrium contract continues to have the form (19). Because cash flows are proportional to the termination payoffs, the EN's payoff in each period is affine in the cash flow over the period. In this generalization, the fixed component of the EN's payoff could be more closely interpreted as a debt payment while the risky component is the equity portion of the EN's payoff for the period.
} 


\section{Equilibrium Dynamics}

We now investigate the properties of the equilibrium contract between the VC and the EN. Before analyzing the general scenario with asymmetric beliefs, we briefly discuss two benchmark scenarios.

\subsection{Symmetric Attitudes towards Risk and Symmetric Beliefs about Project Quality-No Agency}

In this scenario, the $\mathrm{VC}$ and the EN are both risk-neutral and have symmetric beliefs about the project's quality, i.e., $\lambda=0$ and $\Delta_{t}=0$ for all $t$. It follows that the rent from the EN's optimism and the cost of risk components of the VC's periodic flow function (36) are zero. The third component, the return on investment, is always maximized at $b=1$ (Proposition 1). Therefore, the equilibrium pay-performance sensitivities, the VC's investment rates and the EN's effort are constant through time, and the VC's investment rate is at its highest possible level. These results follow from the fact that as the VC and the EN have symmetric attitudes towards risk and symmetric beliefs, they effectively function as a monolithic agent. Moreover, the risk-neutrality of the VC/EN implies that the risk (intrinsic and transient) of the project does not affect the investment rate, the EN's contract or his effort.

\subsection{Symmetric Beliefs}

In this scenario, the VC's periodic flow function

$$
F_{t}(b)=F(b):=-\lambda s b+K c(b)
$$

is independent of time. It is also strictly concave by Proposition 1. The time paths of pay performance sensitivity, investment and effort are all constant; we let $b_{p}^{*}, c_{p}^{*}$ and $\eta_{p}^{*}$ denote the corresponding equilibrium values.

By Proposition 1 the optimal investment function achieves its maximum at $b=1$, which implies that $c^{\prime}(1)=0$. It then follows from $(38)$ that $F^{\prime}(1)<0$. Since $F^{\prime}\left(b_{p}^{*}\right)=0$, the strict concavity of $F(\cdot)$ now guarantees that $b_{p}^{*}<1$. Both $c_{p}^{*}$ and $\eta_{p}^{*}$, therefore, are less than the investment rate and effort levels in the "no agency" scenario where the VC and the EN are both risk-neutral and have symmetric beliefs about project quality.

\subsection{Imperfect Information and Asymmetric Beliefs-The Actual Scenario}

In the actual scenario, the VC's periodic flow function may be expressed as

$$
F_{t}(b)=\frac{\Delta_{0}}{\sigma_{0}^{2}} \sigma_{t}^{2} b+F(b) .
$$


Since $\sigma_{t} \rightarrow 0$, it follows from the Theorem of the Maximum that $b_{t}^{*} \rightarrow b_{p}^{*}$, and thus $\left(c_{t}^{*}, \eta_{t}^{*}\right) \rightarrow\left(c_{p}^{*}, \eta_{p}^{*}\right)$ by continuity where $\left(b_{p}^{*}, c_{p}^{*}, \eta_{p}^{*}\right)$ are the equilibrium pay-performance sensitivity, investment rate, and effort in the benchmark scenario with symmetric beliefs discussed in the previous subsection. We now characterize the dynamics of these economic variables (conditional on the project's continuation)..$^{13}$

\section{Theorem 2 (The Dynamics of the Equilibrium)}

Conditional on the project surviving beyond date $t$, the EN's pay-performance sensitivity, $b_{t}^{*}$, the $V C$ 's investment rate, $c_{t}^{*}$, and the EN's effort, $\eta_{t}^{*}$, all decrease monotonically with $t$ and respectively approach $b_{p}^{*}, c_{p}^{*}$ and $\eta_{p}^{*}$ as $t \rightarrow \infty$.

The results of Theorem 2 hinge on the interplay among the value-enhancing effort by the EN that is positively affected by his optimism, the costs of risk-sharing due to the EN's risk aversion that are negatively affected by the project's intrinsic risk, and the effect of both the VC's physical capital investment and the EN's effort on output. Since the EN is optimistic, he is willing to accept a greater portion of the project's risk so that his pay performance sensitivity and effort are initially high. The passage of time lowers the degree of EN optimism as he revises his initial assessment of project quality. The EN's pay performance sensitivity and effort, therefore, decline over time. The decreasing effort of the EN makes it optimal for the $\mathrm{VC}$ to also lower her capital investments.

\subsection{Sensitivity of Equilibrium Dynamics}

Theorem 3 below characterizes how the equilibrium paths of pay performance sensitivity, investment rates and EN's effort are affected by changes to underlying parameters.

\section{Theorem 3 (Sensitivity of Equilibrium Dynamics)}

The paths of the EN's pay performance sensitivity, the VC's investment rates and the EN's effort are each pointwise (a) decreasing functions of the EN's cost of risk $\lambda$; (b) decreasing functions of the initial transient risk $\sigma_{0}$; (c) decreasing functions of the intrinsic risk $s$; (d) increasing functions of the initial degree of asymmetry of beliefs $\Delta_{0}$; and (e) decreasing functions of the EN's cost of effort $k$.

The EN's pay performance sensitivity declines with his cost of risk because an increase in the EN's cost of bearing risk increases the costs of risk-sharing between the $\mathrm{VC}$ and the EN. An increase in the transient risk lowers the degree of asymmetry in beliefs at each date because the "signal to noise ratio" is increased so that the EN "learns faster". Hence, the economic rents to the VC in each period from the EN's optimism are lowered relative to the costs of risk-sharing so that the

\footnotetext{
${ }^{13}$ In Section 5 we show that the termination time is a random stopping time.
} 
EN's pay-performance sensitivity declines. An increase in the intrinsic risk, on the other hand, increases the degree of asymmetry in beliefs at each date because the EN "learns more slowly" but also increases the costs of risk-sharing. Under Assumption (2), the costs of risk-sharing outweigh the benefits of the EN's optimism so that the EN's pay-performance sensitivity also decreases with intrinsic risk. As a consequence, the investment rates and EN's efforts also decline.

An increase in the EN's optimism leads to a corresponding increase in the economic rents to the $\mathrm{VC}$ in each period. The VC exploits this in each period by increasing the pay-performance sensitivity and investment, thereby leading to an increase in effort by the EN. Under Assumption 1, the economic rents the VC can potentially capture due to the EN's exaggerated assessment of project quality are high compared with the costs of risk-sharing and inducing effort from the EN.

\section{Project Duration}

In this section, we investigate the optimal termination decision of the $\mathrm{VC}$, which determines the project's duration. At any date $t$, we show that the VC's continuation value is an increasing, lower semi-continuous function of her current assessment, $\mu_{t}^{V C}$, of the project's quality. Since the VC continues the project if and only if her continuation value is positive, there exists a trigger level at each date such that she continues the project if and only if her current assessment of the project's quality exceeds the trigger.

\section{Proposition 2}

The optimal stopping policy for the $V C$ is a trigger policy: there exist $\mu_{t}^{*}$ such that the VC continues the project if and only if $\mu_{t}^{V C}>\mu_{t}^{*}$.

Let $Y_{t}^{*} d t:=\left(c_{t}^{* \alpha} \eta_{t}^{* \beta}-0.5 s^{2}-\ell_{t}\right) d t$. Since $d \ln V_{t}=Y_{t}^{*} d t+\xi_{t} d t$ by $(2)$, it follows that

$$
\ln V_{t}-\ln V_{0}=\int_{0}^{t} d \ln V_{u}=\left(\int_{0}^{t} Y_{u}^{*} d u\right)+\left(\int_{0}^{t} \xi_{u} d u\right) .
$$

Given the formula for $\mu_{t}$ given in (4), we may conclude that

$$
\mu_{t} \geq \mu_{t}^{*} \text { if and only if } V_{t} \geq V_{t}^{*}
$$

where

$$
V_{t}^{*}:=V_{0} \exp \left[\left(\int_{0}^{t} Y_{u}^{*} d u\right)+\frac{\left(s^{2}+t \sigma_{0}^{2}\right) \mu_{t}^{*}-s^{2} \mu_{0}}{\sigma_{0}^{2}}\right] .
$$

The process $\left\{V_{t}^{*}\right\}$ represent the performance targets that the project must meet at each date to ensure the continuation of the relationship. Thus, either the $\mu_{t}^{*}$ or the $V_{t}^{*}$ may be used to define the VC's termination policy; the performance targets are more commonly used in practice. 
An increase in the EN's initial degree of optimism about project quality increases the rents that the $\mathrm{VC}$ is able to extract by exploiting the EN's optimism thereby increasing her expected continuation value at each point in time. Hence, it is optimal for the VC to prolong the project's duration. An increase in the EN's cost of risk or cost of effort, however, increases the costs of risk-sharing for the $\mathrm{VC}$, thereby lowering her continuation value at each point in time. Hence, the VC terminates the project earlier. The following result summarizes the effect of the EN's initial assessment of project quality, his cost of risk, and his cost of effort on the duration of the project.

\section{Proposition 3}

The project duration $\tau$ increases with the initial degree of asymmetry in beliefs, decreases with the EN's cost of risk, and decreases with the EN's cost of effort.

The following result establishes that the project is terminated in finite time almost surely. The result ensures that our assumption of a finite time horizon for the VC-EN relationship does not entail a significant loss of generality.

\section{Proposition 4}

For any $\delta>0$ there exists a $T>0$ such that if the maximum possible time horizon is $T^{\prime} \geq T$, then the project duration is strictly less than $T$ with probability greater than $1-\delta$.

\section{Numerical Implementation and Calibration}

We have developed a structural model of venture capital investment and described static and dynamic properties of the equilibrium. Since there is no closed-form analytical characterization of the optimal termination time described by Proposition 2, we use numerical simulation to explore further implications of the model, including comparative static analyses. We use a discrete-time approximation of the continuous-time model whose numerical implementation proceeds as follows. We directly model the evolution of the VC's current assessment of project quality $\mu_{t}^{V C}$ (see 5) because, as explained in Section 5, it determines her continuation decision at any date $t$. In the first stage, we approximate the evolution of $\mu_{t}^{V C}$ using a discrete lattice and derive the termination triggers $\mu_{t}^{*}$. In the second stage, given the triggers obtained from the first stage, we use Monte Carlo simulation to model the evolution of $\mu_{t}^{V C}$ and to obtain the key output variables of interest. ${ }^{14}$ Since Gompers (1995) reports that the average length of a round of VC financing is approximately one year, we set the time period between successive dates in the discrete lattice to one year and assume that it corresponds to a single round of financing. ${ }^{15}$

\footnotetext{
${ }^{14}$ The details of the numerical implementation are available upon request.

${ }^{15}$ We abstract from the possible staging of investments within a particular round of financing.
} 
We classify the parameters into two groups: "direct" parameters whose baseline values can be set using guidance from previous empirical research, and "indirect" parameters whose values are estimated by matching statistics predicted by the model to their observed values in the data. We assume a production technology with constant returns to scale so that $\beta=1-\alpha$. Cochrane (2005) reports an average risk-free rate of 0.068 and finds that the average return on venture capital investment in his sample is $15 \%$. Accordingly, we set the risk-free rate $r$ to 0.068 and the benchmark return $R_{b}$ to $15 \%$. We assume a quadratic form $l(t)=\ell_{1} t^{\ell_{2}}$ for the loss function. With this functional form, there are 11 parameters of the model, grouped into the "technology", "preference" and "belief" categories (see Table 1), whose baseline values we estimate by matching the model's predictions to the data.

In our estimation, we use a collection of 19 aggregate statistics on the distributions of investments, payoffs, risks and returns of venture capital projects reported in previous empirical research. Sahlman (1990, Figure 1) reports eleven statistics describing the distributions of payoffs and investments for a sample of 383 venture capital projects. He reports the termination value and investment (as proportions of the total termination value and investment for all the projects) for projects whose returns are negative, between zero and two times their investment, between two and five times their investment, between five and ten times their investment, and greater than ten times their investment. He also reports the ratio of the total termination value to the total investment for all projects in the sample. ${ }^{16}$ In addition to the Sahlman (1990) statistics, we also use statistics on the risks and returns of venture capital projects reported in Cochrane (2005, Table 4) - specifically, the returns and variances of the round-by-round returns of VC projects in each of the first four rounds of financing. ${ }^{17}$ The 19 statistics used for our estimation are displayed in the first rows of the two panels of Table 2 .

We use the simulated method of moments to estimate the values of the 11 indirect parameters of the model by matching the predicted values of the 19 statistics in Table 2 to their observed values. The standard errors of the estimates are determined by parametric bootstrapping (see Davison and Hinkley, 1997). As shown in Table 2, the model is able to closely match the observed statistics; the

\footnotetext{
${ }^{16}$ The proxies for the Sahlman statistics in our model are obtained as follows. Let Inv $:=V_{0}+\sum_{t=0}^{\tau} c_{t}^{*} V_{t}$ denote the cumulative investment in a project and let Ret $:=V_{\tau} /$ Inv represent the total return on investment. The Sahlman statistics describing the project's payoffs and investments in different regions of the distribution of returns are

$$
\frac{E\left[V_{\tau} 1\left(\operatorname{Ret} \in \mathcal{I}_{j}\right)\right]}{E\left[V_{\tau}\right]} ; \quad \frac{E\left[\operatorname{Inv} 1\left(\operatorname{Ret} \in \mathcal{I}_{j}\right)\right]}{E[\operatorname{Inv}]}, j=1,2,3,4,5,
$$

where $\mathcal{I}_{1}=(-\infty, 0], \mathcal{I}_{2}=(0,2], \mathcal{I}_{3}=[2,5), \mathcal{I}_{4}=[5,10), \mathcal{I}_{5}=[10, \infty)$. For the same sample, Sahlman also computes $E\left[V_{\tau} /\right.$ Inv $]$, the average value per unit of investment.

${ }^{17}$ Because the time period between successive dates in the discrete lattice is one year, which corresponds to the length of a single round of financing, the round-by-round returns of a VC project in the model are $\ln \frac{V_{t}}{V_{t-1}+c_{t-1} V_{t-1}}$, $1 \leq t \leq 4$.
} 
key "belief parameters", in particular, are estimated quite accurately.

The estimated value of the degree of asymmetry in beliefs $\Delta_{0}=0.2457$ is almost four times the VC's assessment of the mean project quality $\mu_{0}^{V C}=0.0660$. The data, therefore, suggest that the level of entrepreneurial optimism is very significant. In the next section, we confirm the substantial impact of entrepreneurial optimism on the characteristics of venture capital relationships. The baseline values of the average intrinsic risk $s$ and transient risk $\sigma_{0}$ are high, which confirms anecdotal and empirical evidence that venture capital is risky and is characterized by significant uncertainty about project quality.

\section{Numerical Analysis}

To explore the impact of EN optimism, we first analyze the model when the parameters take their baseline values in Table 1 . We then explore various comparative static relationships. In our numerical analyses, we compare the actual scenario in which there are asymmetric beliefs and agency conflicts with the two benchmark scenarios discussed in Section 4, namely, the no agency and symmetric beliefs scenarios.

We compute three key output variables in each of the three scenarios. In what follows we interpret the benchmark return on assets $R_{b}$ as the project's discount rate and use it to value the project's cash flows. The Project Value (at date zero) is the expected total payoffs to the firm less the capital investments discounted at the rate $R_{b}$, and is given by

$$
\text { Project Value }:=E_{0}^{V C}\left[e^{-R_{b} \tau} V_{\tau}-\sum_{t=0}^{\tau-1} e^{-R_{b} t} c_{t} V_{t}\right] \text {. }
$$

The VC Fund Stake (at date zero) is the project value less the termination payoff to the EN discounted at the rate $R_{b}$, and is given by

$$
\text { VC Fund Stake }:=\text { Project Value }-E_{0}^{V C}\left[e^{-R_{b} \tau} P_{\tau}\right]
$$

We also compute the total discounted investment (at date zero) in the project, which is given by

$$
\text { Investment }=E_{0}^{V C}\left[\sum_{t=0}^{\tau-1} e^{-R_{b} t} c_{t} V_{t}\right] .
$$

The expectations in (40)-(42) are with respect to the VC's beliefs about project quality, which are assumed to be correct. The termination payoff process evolves as in (1) with the contractual parameters, $\left(a^{*}, b^{*}, c^{*}\right)$, the EN's effort, $\eta^{*}$, and the performance targets, $V^{*}$, set to their equilibrium values for the specific economic scenario (no agency, symmetric beliefs or actual) being analyzed. 


\subsection{Baseline Analysis}

Table 1 reports the Project Value, VC Fund Stake, and Investment as defined above for the baseline model in the actual scenario and the two benchmark scenarios. The difference between the project values (VC fund stakes) in the no agency and symmetric benchmark scenarios could be viewed as the deadweight agency costs of risk sharing between the VC and EN from the perspective of the firm (the VC fund). The difference between the project values (VC fund stakes) in the actual and symmetric scenarios reflect the positive economic rents from EN optimism accruing to the firm (the VC fund). EN optimism significantly mitigates the deadweight agency costs of risk-sharing between the $\mathrm{VC}$ and the EN. With respect to project value, EN optimism lowers the agency costs of risk sharing by over $26 \%$. From the perspective of the VC fund, the benefit of EN optimism is even greater. In fact, the VC Fund Stake in the actual scenario exceeds the VC Fund Stake in the no agency scenario by over $21 \%$. The VC exploits the EN's optimism to her advantage and disproportionately increases the VC fund's share of the resulting surplus.

Table 1 also displays the distribution of the project's duration. Here, $p_{i}^{*}:=\operatorname{Pr}\{\tau=i+1\}$. The project has a significantly greater probability $(50.6 \%)$ of being terminated at the end of one year as compared with the no agency scenario (32.7\%). The benefits of EN optimism are, however, reflected when comparing the actual to the symmetric scenario: the project is more likely to last longer and, therefore, generate more value.

Table 3 reports the EN's pay-performance sensitivities and the (proportional) investment rates for the first four rounds. Consistent with Theorem 2, the EN's pay-performance sensitivity and the VC's investment rate decline over time. The EN's pay-performance sensitivity decreases sharply across the four periods, while the proportional investment rates are practically constant. Successive capital infusions by the VC, therefore, rapidly reduce the EN's stake in the firm.

\subsection{Comparative Statics}

We now carry out several "comparative statics" analyses by varying parameters about their baseline values (+/- $50 \%$ in increments of $2.5 \%)$. We explore the comparative statics of the Project Value, the VC Fund Stake, Investment (defined in 40, 41 and 42) and the Expected Project Duration with respect to four parameters of interest: the physical capital intensity of the project's production function, $\alpha$, the initial transient risk, $\sigma_{0}$, the intrinsic risk, $s$, and the initial degree of asymmetry in beliefs, $\Delta_{0}$. 


\section{The Effects of Human and Physical Capital Intensity}

As shown in Figure 1, Project Value, the VC Fund Stake, Investment and Expected Project Duration decline with the project's physical capital intensity. In our numerical analyses, we assume a constant returns-to-scale production function so that $\alpha+\beta=1$. Hence, as the physical capital intensity increases, the human capital intensity decreases, which reduces the relative contribution of the EN's effort to output.

As shown by Table 4, the VC's proportional investment rate in the project in any round (conditional on continuation) increases as the physical capital intensity increases. However, the VC's dollar investment in any round $i$ is $c_{i-1}^{*} V_{i-1}$. As the physical capital intensity increases, the lower surplus generated by the EN's effort causes the termination payoff process to decline path-wise and the termination probabilities to increase, i.e., the project terminates earlier. Hence, even though the proportional investment rates conditional on continuation increase with the physical capital intensity, the total investment over the duration of the project declines. Moreover, consistent with the fact that the relative contribution of the EN's effort declines with the physical capital intensity, the EN's pay-performance sensitivities in each round decline as shown by Table 4 . Because the relative contribution of the EN's effort to output declines with the physical capital intensity, the deadweight agency costs of risk-sharing between the $\mathrm{VC}$ and the risk-averse EN also decline. The above results lead to the following testable implications:

\section{Testable Implications 1}

a) Project Value, the VC Fund Stake, Investment and Expected Project Duration decrease with the physical capital intensity of the project and increase with its human capital intensity.

b) The EN's pay-performance sensitivity in any round decreases with the project's physical capital intensity and increases with its human capital intensity. The VC's proportional investment rate decreases with the project's physical capital intensity and increases with its human capital intensity.

\section{The Effects of Transient Risk}

An increase in the transient risk has potentially opposing effects on Project Value, the VC Fund Stake, Investment and Expected Project Duration. On the one hand, the EN "learns more quickly" due to an increase in the "signal to noise" ratio (see 7). The faster decline of the EN's optimism lowers the economic rents that the VC could extract from the EN's optimism. On the other hand, an increase in the transient risk increases the likelihood of both high and low realizations of project quality. Since the VC can limit her downside by terminating the relationship if intermediate signals of project quality are sufficiently poor, the option value of the project increases. As we see 
in Figure 2, the positive effect of the option value dominates the negative effect on the power of incentives to the EN, thereby leading to a sharp increase in the Project Value, the VC Fund Stake and Investment. The Expected Project Duration, however, is non-monotonic: it decreases with transient risk below a threshold and then increases.

Table 4 further illustrates the positive effect of the option value of continuation. The probability of the project being continued beyond the third round dramatically increases. (It also shows that with a higher likelihood of a low realization, the probability of terminating at the end of round 1 increases.) The EN's pay-performance sensitivity in any round declines with transient risk, while the VC's proportional investment rates are almost constant.

Consistent with the evidence in Cochrane (2005), Figure 2 reflects the highly skewed nature of the payoffs from venture capital investments and the tremendous value that could be generated by the presence of uncertainty about the quality of innovative ventures. The increase in the VC Fund Stake with transient risk is consistent with empirical evidence that VCs have significant incentives to finance highly innovative projects compared with mature or "imitating" projects because there is greater uncertainty about the quality of innovative projects (Hellman and Puri, 2000).

The above results lead to the following testable implications:

\section{Testable Implications 2}

a) Project Value, the VC Fund Stake and Investment increase with the project's transient risk, while the Expected Project Duration varies non-monotonically in a $U$-shaped manner.

b) The EN's pay-performance sensitivity in any round declines with the project's transient risk, while the VC's proportional investment rate does not vary significantly.

\section{The Effect of Intrinsic Risk}

The benchmark return $R_{b}$ can vary with intrinsic risk. For concreteness, we assume that the benchmark return is the following affine function of the intrinsic risk:

$$
R_{b}(s):=r+\frac{\hat{R}_{b}-r}{\hat{s}} s
$$

where $\hat{R}_{b}$ and $\hat{s}$ denote the baseline values of the benchmark return and the intrinsic risk reported in Table 1 and $r$ is the risk-free rate set to 0.068 as reported in Cochrane (2005).

The effects of intrinsic risk are not a priori obvious because an increase in intrinsic risk has several competing effects. First, in contrast to the effect of transient risk, an increase in the intrinsic risk causes the EN to "learn more slowly" because the "signal to noise" ratio decreases (see 7). The slower convergence of the EN's beliefs has a positive effect on the rents the VC is able to extract from the EN's optimism. Second, an increase in the intrinsic risk increases the costs of risk sharing 
between the VC and the EN, which has a negative effect on Project Value, VC Fund Stake, and Investment. Third, the benchmark return $R_{b}(s)$ decreases with intrinsic risk, which has a negative effect on Project Value and the VC Fund Stake. Fourth, the effect of intrinsic risk on the option value of continuing the relationship is non-monotonic. The standard deviation of the assessment of project quality, $\sigma_{t}^{\mu}$, given by (6) decreases with the intrinsic risk before time $t(s):=\left(s / \sigma_{0}\right)^{2}$ and increases with the intrinsic risk after this time. ${ }^{18}$ Since $t(s)$ increases with $s$, an increase in $s^{2}$ delays the benefits of the option value of continuing the relationship.

As shown in Figure 3, Project Value, the VC Fund Stake, Investment and the Expected Project Duration decline with intrinsic risk. The increase in the cost of risk, the lower economic rents and the higher discount rate outweigh the potential increases in the option value. These results lead to the following testable implications:

\section{Testable Implications 3}

a) Project Value, VC Fund Stake, Investment and Expected Project Duration all decrease with the project's intrinsic risk.

b) The EN's pay-performance sensitivity in any round decreases with the project's intrinsic risk, while the VC's proportional investment rate does not vary significantly.

\section{The Effect of the Degree of Asymmetry in Beliefs}

The increase in Project Value, VC Fund Stake, Investment and the Expected Project Duration with the degree of asymmetry in beliefs shown in Figure 4 illustrate the positive effects of the EN's optimism. An increase in the degree of asymmetry in beliefs mitigates the costs of risk sharing between the $\mathrm{VC}$ and the EN thereby enhancing the power of incentives that could be provided by the EN. As shown by Table 4, the EN's pay-performance sensitivity in any round increases with the degree of asymmetry in beliefs, but the VC's proportional investment rate is relatively insensitive. These results lead to the following testable implications:

\section{Testable Implications 4}

a) Project Value, VC Fund Stake, Investment and Expected Project Duration all increase with the initial degree of asymmetry in beliefs about project quality.

b) The EN's pay-performance sensitivity in any round increases with the initial degree of EN optimism, while the VC's proportional investment rate does not vary significantly.

Previously reported empirical evidence is indirectly consistent with the implication a) above. The positive effects of EN optimism on project value are consistent with the evidence reported in

\footnotetext{
${ }^{18}$ Follows directly from $d \sigma_{t}^{\mu} / d s=\sigma_{0}^{2}\left(t \sigma_{0}^{2}-s^{2}\right) /\left(s^{2}+t \sigma_{0}^{2}\right)^{2}$.
} 
Gelderen et al (2005). The positive relation between duration and the degree of EN optimism is consistent with the evidence in Kaplan and Stromberg (2003) that experienced entrepreneurs, who are likely to have more realistic beliefs, receive fewer rounds of financing.

\subsection{Implied Discount Rate and Optimism Ratio}

There is considerable empirical and anecdotal evidence that VCs typically use high discount rates in the range between $35 \%$ and 50\% to value projects (see Sahlman, 1990, Gladstone and Gladstone, 2002). Sahlman (1990) suggests that high discount rates could be a mechanism that VCs use to adjust optimistic projections by ENs. We now explore whether the levels of EN optimism predicted by the model could lead to the discount rates observed in reality. We calculate the implied discount rate (IDR) of a project as the discount rate the $\mathrm{VC}$ would use to obtain her valuation of the fund's stake defined in (41) if the project's intrinsic quality were (hypothetically) distributed according to the EN's beliefs. More precisely, the implied discount rate $\beta_{V C}$ solves:

$$
E_{0}\left[e^{-\beta_{V C} \tau}\left(V_{\tau}-P_{\tau}\right)-\sum_{t=0}^{\tau-1} e^{-\beta_{V C} t} c_{t} V_{t} \mid \Theta \sim N\left(\mu_{0}^{E N}, \sigma_{0}^{2}\right)\right]=\text { VC Fund Stake. }
$$

Figure 5 reports the IDR's for varying values of $\alpha, \sigma_{0}, s, \Delta_{0}$. Except for the scenario where $\Delta_{0}$ varies, all IDR's lie in a very narrow range around $40 \%$. These values for the IDR's are consistent with the discount rates reported in Table 6 of Sahlman (1990) that VC's use to assess the value of a new venture. Because $\Delta_{0}$ is, in effect, the entrepreneurial optimism premium, the variation of the IDR's with $\Delta_{0}$ is approximately linear. The implied discount rates are much higher than the average return on VC projects reported in Cochrane (2005) because they adjust for the EN's optimistic projections of the project's payoffs.

The fact that our model, which is calibrated to the data on the distributions of investments and payoffs in Sahlman (1990) and VC projects' risk and return data in Cochrane (2005), generates implied discount rates consistent with those reported in Sahlman (1990) strongly suggests that entrepreneurial optimism, in fact, explains the discount rates used by VCs in reality. Moreover, the numerical results suggest that an IDR of $40 \%$ is a remarkably good rule-of-thumb to value the average venture capital project with entrepreneurial projections of payoffs.

An alternative to using higher discount rates to adjust optimistic EN cash flow projections is to simply lower the cash flow projections and discount the cash flows at the usual discount rate to value projects. Gladstone and Gladstone (2002, p. 93) report that, on average, VCs halve EN payoff projections so that the EN Optimism Ratio is approximately two on average. In our model, 
the Optimism Ratio is given by

$$
\begin{aligned}
\text { Optimism Ratio: } & =\frac{\text { VC Fund Stake under EN Beliefs }}{\text { VC Fund Stake under VC Beliefs }} \\
& =\frac{E_{\Theta=N\left(\mu_{0}^{E N}, \sigma_{0}^{2}\right)}\left[e^{-R_{b} \tau^{E N}}\left(V_{\tau}-P_{\tau}\right)-\int_{0}^{\tau^{E N}} e^{-R_{b} u} c_{u} V_{u} d u\right]}{E_{\Theta=N\left(\mu_{0}^{V C}, \sigma_{0}^{2}\right)}\left[e^{-R_{b} \tau}\left(V_{\tau}-P_{\tau}\right)-\int_{0}^{\tau} e^{-R_{b} u} c_{u} V_{u} d u\right]} .
\end{aligned}
$$

In the numerator (denominator) on the right-hand side of the second equality above, the project's intrinsic quality $\Theta$ is distributed according to the EN's (VC's) prior and its termination payoff and investments are discounted at the benchmark rate $R_{b}$. The superscript on the termination time $\tau^{E N}$ in the numerator emphasizes that the project's termination time (in the hypothetical scenario in which its intrinsic quality is distributed according to the EN's beliefs) differs from the termination time in the actual scenario (where intrinsic quality is distributed according to the VC's beliefs).

Figure 6 shows the variation of the Optimism Ratio with $\alpha, \sigma_{0}, s, \Delta_{0}$. The Optimism Ratios mostly range between 2 and 3 . When the parameters take their baseline values, the Optimism Ratio is 2.36. The range of Optimism Ratios predicted by the model are consistent with the anecdotal evidence in Gladstone and Gladstone (2002, p. 93) that the Optimism Ratio is approximately 2.

Not surprisingly, the Optimism Ratio increases with the degree of EN optimism. The effects of transient risk, intrinsic risk and capital intensity on the Optimism Ratio are more subtle. By the intuition for Figure 2, an increase in the transient risk increases the project value because of the increase in the option value of continuing the relationship. Because the likelihood of negative signals that force early termination is significantly lower, the increase in the option value when the project value is (hypothetically) distributed according to the EN's optimism beliefs is greater relative to the actual scenario where it is distributed according to the VC's beliefs. Hence, an increase in the transient risk increases the numerator of (45) to a greater extent than the denominator so that the Optimism Ratio increases. As discussed in the intuition for Figure 3, a decrease in the intrinsic risk decreases the costs of risk sharing and generally increases the option value of continuation. The positive effects of a decrease in the intrinsic risk are relatively greater when the project quality is (hypothetically) distributed according to the EN's beliefs so that the Optimism Ratio increases with a decrease in the intrinsic risk. By the intuition for Figure 1, a decrease in the physical capital intensity is accompanied by an increase in the human capital intensity that raises the project value. The positive effects of an increase in the human capital intensity are relatively greater when the project quality is distributed according to the EN's beliefs so that the Optimism Ratio increases with human capital intensity and decreases with physical capital intensity. 


\subsection{Internal Rate of Return}

The venture capital industry typically uses internal rates of return to evaluate projects. In our model, a project's Internal Rate of Return (IRR) is the value $R^{*}$ that solves the following equation:

$$
E_{0}\left[e^{-R^{*} \tau}\left(V_{\tau}-P_{\tau}\right)-\sum_{t=0}^{\tau-1} e^{-R^{*} t} c_{t} V_{t} \mid \Theta \sim N\left(\mu_{0}^{V C}, \sigma_{0}^{2}\right)\right]-V_{0}=0 .
$$

Figure 7 shows the variation of the IRR's. The IRR in the baseline scenario is $58 \%$, which is consistent with the average internal rate of return of venture capital projects reported in Gladstone and Gladstone (2002). By Figure 3, the positive effects of an increase in the transient risk on the VC fund stake are much greater relative to its effects on investment. The IRR, therefore, increases with transient risk. Similarly, as shown in Figures 1 and 3, an increase in the project's physical capital intensity and intrinsic risk has a more pronounced negative effect on the VC fund stake than investment so that the IRR decreases. By Figure 3, the marginal effects of the degree of EN optimism on the VC fund stake and investment are approximately equal so that the IRR is relatively insensitive to EN optimism. The findings of Figure 7 lead to the following testable implications:

\section{Testable Implications 5}

The Internal Rate of Return of a project increases with its transient risk, decreases with its intrinsic risk and physical capital intensity, but does not vary significantly with the degree of EN optimism.

\section{Conclusions}

We develop a dynamic, structural model to obtain quantitative assessments of the effects of entrepreneurial optimism on the characteristics of venture capital relationships. Our principal-agent framework incorporates several key features of VC relationships, namely, the presence of risky payoffs and agency conflicts, uncertainty and asymmetric beliefs about project quality, and the importance of staged investment and dynamic contracting in mitigating potential inefficiencies arising from these imperfections. Consistent with observed contracts, the optimal dynamic contracts between VCs and ENs have "debt" and "equity" components. The EN's stake in the project vests over time, and the VC's claim allows her to recover her investments with high probability. We show that EN optimism is a key determinant of the characteristics of VC relationships - the value that they generate, the pattern of $\mathrm{VC}$ investments over time, and the structure of VC-EN contracts.

We derive empirically testable implications for the effects of projects' intrinsic risk, transient risk, physical and human capital intensity as well as the degree of EN optimism on the duration and economic value of $\mathrm{VC}$ relationships. The interplay among the intrinsic and transient risks of 
projects and the degree of EN optimism leads to significant heterogeneity in contractual structures and the patterns of staged VC investment, which is consistent with empirical evidence. The intrinsic and transient risks of projects have opposite effects on the durations and economic values of VC relationships. The value of the project and the expected payoff to the $\mathrm{VC}$ are actually enhanced when there is greater noise in the perception of project quality.

With significantly fewer free parameters or degrees of freedom, the model is able to closely match a disparate set of statistics on the distributions of investments, payoffs, risks and returns of venture capital projects as well as the discount rates used to value them. In particular, our structural approach suggests that entrepreneurial optimism is, in fact, significant enough to generate the huge discrepancy between discount rates used by VCs to value projects (which adjust for optimistic projections by ENs) and the average expected return of VC projects.

The tractability of the model, coupled with the fact that it is able to match disparate statistics pertaining to the risks and returns of VC projects as well as the discount rates used to value them, suggests that it could be useful as a tool to value risky projects when agents have asymmetric beliefs and have conflicts of interest. Although we focus on venture capital investment, our framework is more generally applicable to other dynamic principal-agent settings with double-sided moral hazard, risk, imperfect information, and asymmetric beliefs. For example, our framework could be applied to study the relationship between the shareholders of a firm and its manager or employees, the financing of research and development, and delegated portfolio management (mutual funds, hedge funds). We explore these applications in future research. 


\section{References}

1. Allen, F. and Gale, D. (1999), "Diversity of Opinion and the Financing of New Technologies," Journal of Financial Intermediation, 8, 68-89.

2. Alvarez, F. and Jermann, U. (2000), "Efficiency, Equilibrium, and Asset Pricing with Risk of Default," Econometrica, 68, 4, 775-797.

3. Baker, M., Ruback, R. and Wurgler, J. (2005), "Behavioral Corporate Finance: A Survey," Handbook of Corporate Finance: Empirical Corporate Finance (ed: E. Eckbo), Part III, Chapter 5, Elsevier/North-Holland.

4. Bergemann, D. and Hege, U. (1998), "Venture Capital Financing, Moral Hazard, and Learning," Journal of Banking \& Finance, 22, 703-735.

5. Berk, J. and Green, R. and Naik, V. (2004), "Valuation and Return Dynamics of New Ventures," The Review of Financial Studies, 17, 1-35.

6. Casamatta, C. (2003), "Financing and Advising: Optimal Financial Contracts with Venture Capitalists," Journal of Finance, 58, 2059-2086.

7. Cochrane, J. (2005), "The Risk and Return of Venture Capital," Journal of Financial Economics, $\mathbf{7 5}, 3-52$.

8. Cornelli, F. and Yosha, O. (2003), "Stage Financing and the Role of Convertible Securities," Review of Economic Studies, 70, 1-32.

9. Cuny, C. and Talmor, E. (2005), "The Staging of Venture Capital Financing: Milestone vs. Round," Working Paper.

10. Davison, A.C. and Hinkley, D.V. (1997). Bootstrap Methods and their Application, Cambridge Series in Statistical and Probabilistic Mathematics.

11. DeMarzo, P. and Fishman, M. (2004), "Optimal Long-Term Financial Contracting with Privately Observed Cash Flows," Working Paper.

12. Dessein, W. (2005), "Information and Control in Ventures and Alliances," Journal of Finance, 60, 2513-2549.

13. Duffie, D. (2001), Dynamic Asset Pricing Theory, Third Edition, Princeton University Press.

14. Duffie, D. and Epstein, L. (1992), "Stochastic Differential Utility," Econometrica, 60, 353-394.

15. Egli, D. and Ongena, S. and Smith, D.C. (2006), "On the Sequencing of Projects, Reputation Building, and Relationship Finance," Finance Research Letters, 3, 23-39.

16. Fudenberg, D., Holmstrom, B. and Milgrom, P. (1990), "Short-Term Contracts and LongTerm Agency Relationships," Journal of Economic Theory, 51, 1-31.

17. Gelderen, M. and Thurik, R. and Bosma, N. (2005), "Success and Risk Factors in the PreStartup Phase," Small Business Economics, 24, 365-380.

18. Gibbons, R. and Murphy, K. (1992), "Optimal Incentive Contracts in the Presence of Career Concerns: Theory and Evidence," Journal of Political Economy, 100, 468-505.

19. Gladstone, D. and Gladstone, L. (2002), Venture Capital Handbook: An Entrepreneur's Guide to Raising Venture Capital, Prentice Hall, Upper Saddle River, New Jersey. 
20. Gompers, P. (1995), "Optimal Investment, Monitoring, and the Staging of Venture Capital," The Journal of Finance, 50, 1461-1489.

21. Gompers, P. and Lerner, J. (1996), "The Use of Covenants: An Empirical Analysis of Venture Partnership Agreements," Journal of Law and Economics, 39, 463-498.

22. Hellmann, T. and Puri, M. (2000), "The Interaction Between Product Market and Financing Strategy: The Role of Venture Capital," The Review of Financial Studies, 13, 959-984.

23. Hellmann, T. and Puri, M. (2002), "Venture Capital and the Professionalization of Start-Up Firms: Empirical Evidence," The Journal of Finance, 57, 169-197.

24. Holmstrom, B. (1999), "Managerial Incentive Problems: A Dynamic Perspective," Review of Economic Studies, 66, 169-182.

25. Holmstrom, B. and Milgrom, P. (1987), "Aggregation and Linearity in the Provision of Intertemporal Incentives," Econometrica, 55, 2, 303-328.

26. Inderst, R. and Muller, H. (2004), "The Effect of Capital Market Characteristics on the Value of Start-Up Firms", Journal of Financial Economics, 72, 319-356.

27. Kaplan, S. and Stromberg, P. (2003), "Financial Contracting Theory Meets the Real World: An Empirical Analysis of Venture Capital Contracts," Review of Economic Studies, 70, 281315 .

28. Kirilenko, A. (2001), "Valuation and Control in Venture Finance," The Journal of Finance, 56, 565-587.

29. Kiyotaki, N. and Moore, J. (1997), "Credit Cycles", Journal of Political Economy, 105, 2, 211-248.

30. Kockesen, L. and Ozerturk, S. (2004), "Exclusivity and Overinvestment: A Model of Relationship Financing," Working Paper, Columbia University.

31. Landier, A. and Thesmar, D. (2005), "Financial Contracting with Optimistic Entrepreneurs: Theory and Evidence," Working Paper.

32. Ma, J. and Yong, J. (1999), Forward-Backward Stochastic Differential Equations and Their Applications, Springer Lecture Notes in Mathematics.

33. Neher, D. (1999), "Staged Financing: An Agency Perspective," Review of Economic Studies, 66, 255-274.

34. Oksendal, B. (2003), Stochastic Differential Equations: An Introduction with Applications, Sixth Edition, Springer-Verlag, Heidelberg, Germany.

35. Repullo, R. and Suarez, J. (2004), "Venture Capital Finance: A Security Design Approach," Review of Finance, 8, 75-108.

36. Sahlman, W. (1990), "The Structure and Governance of Venture-Capital Organizations," Journal of Financial Economics, 27, 473-521.

37. Schmidt, K. (2003), "Convertible Securities and Venture Capital Finance," Journal of Finance, 58, 1139-1166. 


\section{Appendix A}

\section{The Relationship between Outside Investors and the VC}

We present one possible extension of the model in which the VC's compensation structure described in Section 2.3 is endogenously derived. We model the relationship between the VC and outside (existing and potential) investors. At each date, the VC raises capital for her investments. Let $\mathcal{A}_{t}$ denote the total capital the $\mathrm{VC}$ is able to procure at date $t$, which we derive endogenously in the following. The VC has operating $\operatorname{costs} C\left(\mathcal{A}_{t}\right)$, where $C(\cdot)$ is nonnegative, strictly increasing and convex. The VC's compensation in each period is a fixed proportion $f>0$ of the total capital she manages. ${ }^{19}$ The expected excess return on assets before operating costs and the VC's compensation

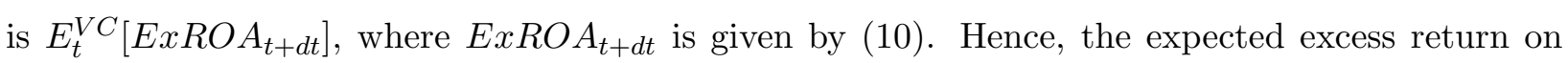
assets net of operating costs and fees or the expected net excess return is

$$
\frac{\mathcal{A}_{t} E_{t}^{V C}\left[E x R O A_{t+d t}\right]-f \mathcal{A}_{t} d t-C\left(\mathcal{A}_{t}\right) d t}{\mathcal{A}_{t}} .
$$

Outside investors competitively supply capital to the VC at each date. In equilibrium, therefore, investors allocate capital until the expected excess return net of operating costs and the VC's compensation is zero. It then follows directly from (47) that, in equilibrium, the capital obtained by the $\mathrm{VC}, \mathcal{A}_{t}^{*}(f)$ (the argument indicates the dependence of the capital raised on the management fee) satisfies

$$
E_{t}^{V C}\left[\operatorname{ExROA} A_{t+d t}\right]=f+\frac{C\left(\mathcal{A}_{t}^{*}(f)\right)}{\mathcal{A}_{t}^{*}(f)} .
$$

Since $C(0)=0$ and $C(\cdot)$ is strictly convex, $C(x)<x C^{\prime}(x)$. Therefore, $\frac{d}{d x} \frac{C(x)}{x}=\frac{x C^{\prime}(x)-C(x)}{x^{2}}>0$ so that $\frac{C(x)}{x}$ is strictly increasing. It then follows from (48) that the capital under management and the VC's compensation are increasing in the expected excess return on assets. If we assume a quadratic operating cost function $C(x)=M x+N x^{2}$ for analytical convenience, the capital obtained by the $\mathrm{VC}$ and her compensation are affine functions of the expected excess return on assets.

\section{The Representation of the EN's Objective as a Recursive Utility}

We show that the EN's objective as described by (13) or (14) actually belong to the general class of recursive or stochastic differential utilities (see Duffie and Epstein, 1992). If

$$
U_{t}=E_{t}^{E N}\left[e^{-r(\tau-t)} P_{\tau}-\int_{t}^{\tau} e^{-r(s-t)} k \eta_{s}^{\gamma} V_{s} d s\right]
$$

\footnotetext{
${ }^{19}$ We can extend the model to allow for the $\mathrm{VC}$ to optimally and dynamically determine the fee at the beginning of each period. As in the simpler setting analyzed here, we can show that the VC's compensation in each period is increasing in the expected excess return on assets so that the main implications of the theory remain unaltered.
} 
is the EN's conditional expected utility at date $t$, then $U_{t}$ satisfies the following backward stochastic differential equation or BSDE (see Ma and Yong, 1999):

$$
\begin{aligned}
d U_{t} & =\left(r U_{t}-k \eta_{t}^{\gamma} V_{t}\right) d t+Z_{t} d B_{t}^{E N}=\left(r U_{t}-k \eta_{t}^{\gamma} V_{t}-\lambda Z_{t}\right) d t+Z_{t} d B_{t} \\
U_{\tau} & =P_{\tau}
\end{aligned}
$$

The second equality above follows from (16). The process $Z_{t}$ is $\left\{\mathcal{F}_{t}\right\}$-adapted and the pair of processes $(U, Z)$ is the solution of the BSDE (50). From (50), the EN's conditional expected utility $U_{t}$ has the general recursive representation

$$
\begin{aligned}
U_{t} & =U_{\tau}+\int_{t}^{\tau} f\left(U_{s}, Z_{s}\right) d s-\int_{t}^{\tau} Z_{s} d B_{s}, \\
f\left(U_{s}, Z_{s}\right) & =-r U_{s}+k \eta_{s}^{\gamma} V_{s}+\lambda Z_{s},
\end{aligned}
$$

where the function $f$ is the aggregator (see Duffie and Epstein, 1992, Ma and Yong, 1999).

\section{Appendix B: Proof of Theorem 1}

A rigorous proof of Theorem 1 requires a precise interpretation of equation (1), which describes the evolution of the termination payoff process. We consider the termination payoff process $V(\cdot)$ to be a given random process on a probability space with investment and effort altering the probability distribution of this process. The uncertainty is represented by a filtered probability space $\left(\Omega, \mathcal{F}, \mathcal{F}_{t}, \mathcal{Q}\right)$ on which is defined a standard Brownian motion $\widehat{B}$. Letting $\left\{\mathcal{F}_{t}^{\widehat{B}}\right\}$ denote the complete and augmented filtration generated by $\widehat{B}$, and $\mathcal{H}$ a $\sigma$-field independent of $\mathcal{F}_{T}^{\widehat{B}}$, the complete information filtration is the augmentation of the filtration $\mathcal{H} \times\left\{\mathcal{F}_{t}^{\widehat{B}}\right\}$. The role of $\mathcal{H}$ is to allow heterogeneity in the VC's and EN's priors. The EN's and VC's beliefs are represented by probability measures $Q^{\ell}, \ell \in\{V C, E N\}$ equivalent to $\mathcal{Q}$, which may disagree on $\mathcal{H}$. $\widehat{B}$ is a Brownian motion under both these probabilities. Consider the process $V(\cdot)$ defined by

$$
d V(t)=s V(t) d \widehat{B}(t)
$$

where $s$ is the intrinsic risk of the project. We use the Girsanov transformation to obtain new probability measures on $(\Omega, \mathcal{F})$ such that the process $V(\cdot)$ evolves as in $(1)$.

$\Theta$ is an $\mathcal{H}$-measurable normal random variable with variance $\sigma_{0}^{2}$ and mean $\mu_{0}^{\ell}$ under measure $Q^{\ell}$. Suppose that $\eta(\cdot)$ and $c(\cdot)$ are strictly positive, $\left\{\mathcal{F}_{t}\right\}$-adapted, square integrable stochastic processes (under the measures $Q^{V C}$ and $Q^{E N}$ ) defined on the time horizon $[0, T]$ describing the EN's choices of effort and the VC's choices of investments over time. Recall that $l(\cdot)$ is a deterministic process 
describing the operating costs of the firm. Define the processes

$$
\begin{array}{r}
\zeta_{c, \eta}^{\ell}(t):=\exp \left[\int_{0}^{t}\left(\Theta+A c(u)^{\alpha} \eta(u)^{\beta}-l(u)-\lambda s 1_{\ell=E N}\right) s^{-1} d \widehat{B}(u)-\right. \\
\left.\frac{1}{2} \int_{0}^{t}\left(\Theta+A c(u)^{\alpha} \eta(u)^{\beta}-l(u)-\lambda s 1_{\ell=E N}\right)^{2} s^{-2} d u\right], \\
B_{c, \eta}^{\ell}(t):=\widehat{B}(t)-\int_{0}^{t}\left(\Theta+A c(u)^{\alpha} \eta(u)^{\beta}-l(u)-\lambda s 1_{\ell=E N}\right) s^{-1} d u .
\end{array}
$$

The process $\zeta_{c, \eta}^{\ell}(\cdot)$ is a positive martingale. ${ }^{20}$ Define the new measure $\Pi_{c, \eta}^{\ell}$ by

$$
\frac{d \Pi_{c, \eta}^{\ell}}{d Q^{\ell}}=\zeta_{c, \eta}^{\ell}(T)
$$

By Girsanov's theorem (see Oksendal, 2003), the process $B_{c, \eta}^{\ell}(\cdot)$ is a Brownian motion under the measure $\Pi_{c, \eta}^{\ell}$. Further, under this measure, the process $V(\cdot)$ evolves as

$$
d V(t)=\left[\Theta+A c(t)^{\alpha} \eta(t)^{\beta}-l(t)-\lambda s 1_{\ell=E N}\right] V(t) d t+s V(t) d B_{c, \eta}^{\ell}(t) .
$$

Equation (56) describes the evolution of the termination payoff process under the actual probability and the EN's subjective valuation probability, and is identical to equations (1) and (15), but with the Brownian motion and the probability measures representing the VC's and EN's beliefs depending on the investment and effort processes. It is important to keep in mind that $V(\cdot)$ is a fixed process whose sample paths are not affected by investment and effort. Investment and effort, however, alter the probability distribution of the sample paths of $V(\cdot)$.

For future reference, we note that the process

$$
d W_{c, \eta}^{\ell}(t):=s^{-1}\left[d \ln V(t)-\left(A c(t)^{\alpha} \eta(t)^{\beta}-0.5 s^{2}-\lambda s 1_{\ell=E N}-l(t)\right) d t-\mu_{t}^{\ell} d t\right]
$$

is an $\left\{\mathcal{F}_{t}\right\}$-Brownian motion with respect to the probability measure $\Pi_{c, \eta}^{\ell}$. Moreover, the complete and augmented filtration generated by this Brownian motion is $\left\{\mathcal{F}_{t}\right\}$. Recall that the EN's and VC's mean assessments of project quality $\Theta$ at date $t, \mu_{t}^{E N}, \mu_{t}^{V C}$ in (2) and (4), do not depend on the effort and investment processes because they are observable. Let $\tau \leq T$ be an $\left\{\mathcal{F}_{t}\right\}$-stopping time denoting the termination time of the VC-EN relationship. Let $c(\cdot), \eta(\cdot)$ and $\widehat{\eta}(\cdot)$ be strictly

${ }^{20}$ The processes are assumed to satisfy the Novikov condition (see Oksendal, 2003):

$$
E^{\ell} \exp \left[\frac{1}{2} \int_{0}^{T}\left(\Theta+A c(u)^{\alpha} \eta(u)^{\beta}-\lambda s 1_{\ell=E N}-l(u)\right)^{2} s^{-2} d u\right]<\infty, \ell \in\{V C, E N\} .
$$

Because the equilibrium investment and effort processes described in Theorem 1 are deterministic and $\Theta$ is a normal random variable, the Novikov condition is satisfied by these processes. In fact, we do not need to assume that feasible (not necessarily optimal) investment and effort processes satisfy the Novikov condition for our analysis to be valid; we only require that they be square-integrable. In this case, the process $\zeta_{c, \eta}(\cdot)$ is only guaranteed to be a local martingale and the measure $\Pi_{c, \eta}^{\ell}$ is a finite measure, but not necessarily a probability measure. Our analysis, however, only requires that $\Pi_{c, \eta}^{\ell}$ be a finite measure. Since the Novikov condition is satisfied by the equilibrium investment and effort processes, the measure corresponding to the equilibrium processes is a probability measure. 
positive $\left\{\mathcal{F}_{t}\right\}$-adapted square-integrable processes on $[0, \tau] .{ }^{21}$ A contract is represented by the triple $(P(\cdot), c(\cdot), \tau)$. The processes below are required in the sequel.

We define the cumulative value process of the EN

$$
\bar{U}_{P, c, \tau}(\eta(\cdot) ; t):=E_{c, \eta}^{E N}\left[e^{-r(\tau-t)} P(\tau)-\int_{0}^{\tau} e^{-r(u-t)} k \eta(u)^{\gamma} V(u) d u \mid \mathcal{F}_{t}\right],
$$

which is the EN's conditional valuation of his future payoffs at any date including the sunk disutilities of prior effort from a given contract $(P(\cdot), c(\cdot), \tau)$ when his effort choices over time are given by the process $\eta(\cdot)$. Here, $E_{c, \eta}^{\ell}\left[\cdot \mid \mathcal{F}_{t}\right] ; \ell \in\{V C, E N\}$ denotes conditional expectation at date $t$ under the probability measure $\Pi_{c, \eta}^{\ell}$ defined in (55). We note that the discounted cumulative value process of the EN, $e^{-r t} \bar{U}_{P, c, \tau}(\eta(\cdot) ; t)$, is a square-integrable $\left\{\mathcal{F}_{t}\right\}$-martingale under the measure $\Pi_{c, \eta}^{E N}$.

Given a contract $(P(\cdot), c(\cdot), \tau)$ and effort process $\eta(\cdot)$, the EN's certainty equivalent process is

$$
R_{P, c, \tau}(\eta(\cdot) ; t):=\bar{U}_{(P, c, \tau)}(\eta(\cdot) ; t)+\int_{0}^{t} e^{-r(u-t)} k \eta(u)^{\gamma} V(u) d u, t \in[0, \tau]
$$

The EN's adjusted cumulative value process represents the cumulative value process of the EN when he exerts effort $\eta(s) ; s \leq t$ and effort $\widehat{\eta}(s) ; s \geq t$. Formally, we define

$$
\begin{gathered}
Y_{P, c, \tau}(\eta(\cdot) ; t ; \widehat{\eta}(\cdot)):= \\
E_{c, \eta}^{E N}\left[e^{-r(\tau-t)} P(\tau)-\int_{0}^{t} e^{-r(u-t)} k \eta(u)^{\gamma} V(u) d u-\int_{t}^{\tau} e^{-r(u-t)} k \widehat{\eta}(u)^{\gamma} V(u) d u \mid \mathcal{F}_{t}\right] .
\end{gathered}
$$

The EN's maximum conditional valuation process represents the EN's maximum conditional valuation of his future payoffs at date $t$ given that he has exerted effort $\eta(s) ; s \leq t$ and the contract is $(P(\cdot), c(\cdot), \tau)$. Formally, we define

$$
Z_{P, c, \tau}(\eta(\cdot) ; t):=\sup _{\widehat{\eta}(\cdot)} Y_{P, c, \tau}(\eta(\cdot) ; t ; \widehat{\eta}(\cdot))
$$

\section{Implementation of a Given EN Effort Process}

To simplify the subsequent notation, we drop the subscripts denoting the dependence of the processes defined in (58)-(61) on the contract $(P(\cdot), c(\cdot), \tau)$. A contract $(P(\cdot), c(\cdot), \tau)$ is said to $\mathrm{im}$ plement a given effort process $\eta^{*}(\cdot)$ if and only if $P(0)=V(0)$ and, given the contract $(P(\cdot), c(\cdot), \tau)$, the EN's optimal effort choices are given by the process $\eta^{*}(\cdot)$. The following Lemma characterizes the contract $(P(\cdot), c(\cdot), \tau)$ that implements a given effort process $\eta^{*}(\cdot)$ of the EN.

\section{Lemma 1}

a) A contract $(P(\cdot), c(\cdot), \tau)$ implements $\eta^{*}(\cdot)$ only if $P(0)=V(0), P(t)=R\left(\eta^{*}(\cdot) ; t\right)$ a.s., and the certainty equivalent process $R\left(\eta^{*}(\cdot) ; \cdot\right)$ satisfies the following stochastic differential equation:

$$
d R\left(\eta^{*}(\cdot) ; t\right)=a(t) V(t) d t+b(t) d V(t),
$$

\footnotetext{
${ }^{21}$ These processes are assumed to satisfy the Novikov condition-see footnote 20.
} 
where

$$
\begin{gathered}
b(t)=\frac{\gamma k}{A \beta c(t)^{\alpha}} \eta^{*}(t)^{\frac{\gamma-\beta}{\beta}}, \\
a(t):=k \eta^{*}(t)^{\gamma}-b(t)\left(A c(t)^{\alpha} \eta^{*}(t)^{\beta}-\lambda s-l(t)+\mu_{t}^{E N}\right)+r \frac{P_{t}}{V_{t}} .
\end{gathered}
$$

b) The EN's continuation value defined in (17) is zero at each date.

Proof. a) When the certainty equivalent process $R\left(\eta^{*}(\cdot) ; t\right)$ corresponding to the given effort process $\eta^{*}(\cdot)$ evolves as in (62), we show below that the EN's optimal effort choices after any given date $t$ coincide with the process $\eta^{*}(\cdot)$ regardless of his prior history of effort choices. It will then follow that the process $\eta^{*}(\cdot)$ describes the EN's optimal effort choices over the entire interval $[0, \tau)$ so that the contract $(P(\cdot), c(\cdot), \tau)$ implements the given effort process $\eta^{*}(\cdot)$.

By the principle of optimality of dynamic programming (Oksendal, 2003), the effort $\eta^{*}(t)$ is optimal for the EN at date $t$ for any prior effort process $\eta(\cdot)$ only if ${ }^{22}$

$$
\begin{aligned}
\eta^{*}(t) & =\operatorname{argmax}_{\eta(t)} E_{c, \eta}^{E N}\left[e^{-r d t} Z(\eta(\cdot) ; t+d t)-Z(\eta(\cdot) ; t) \mid \mathcal{F}_{t}\right] \\
& =\operatorname{argmax}_{\eta(t)} E_{c, \eta}^{E N}\left[d Z(\eta(\cdot) ; t)-r Z(\eta(\cdot), t) d t \mid \mathcal{F}_{t}\right] .
\end{aligned}
$$

In what follows, we derive the infinitesimal change $d Z(\eta(\cdot) ; t)$ and then use (65) to establish the statements of the Lemma. It follows from the definition (61) that

$$
\begin{aligned}
Z(\eta(\cdot) ; t) & =\sup _{\eta^{\prime}(\cdot)} Y\left(\eta(\cdot) ; t ; \eta^{\prime}(\cdot)\right) \\
& =\sup _{\eta^{\prime}(\cdot)} Y\left(\eta^{*}(\cdot) ; t ; \eta^{\prime}(\cdot)\right)+k \int_{0}^{t} e^{-r(s-t)}\left[\eta(s)^{\gamma}-\eta^{*}(s)^{\gamma}\right] V(s) d s \\
& =Z\left(\eta^{*}(\cdot) ; t\right)+X(\eta(\cdot) ; t),
\end{aligned}
$$

where we define the stochastic process

$$
X(\eta(\cdot) ; t):=k \int_{0}^{t} e^{-r(s-t)}\left[\eta(s)^{\gamma}-\eta^{*}(s)^{\gamma}\right] V(s) d s .
$$

The second equality in (66) follows because the contract $P($.$) only depends on the history of the$ termination payoff process, and because the EN's effort choices are observable. Hence, his prior effort choices over the interval $[0, t]$ do not affect his optimal effort choices over the interval $[t, \tau]$. It may be readily verified from (66) and (67) that

$$
d Z(\eta(\cdot) ; t)=d Z\left(\eta^{*}(\cdot) ; t\right)+k\left(\eta(t)^{\gamma}-\eta^{*}(t)^{\gamma}\right) V(t) d t+r X(\eta(\cdot), t) d t
$$

Since the process $\eta^{*}(\cdot)$ represents the EN's optimal effort choices by hypothesis, it follows that

$$
Z\left(\eta^{*}(\cdot), t\right)=\bar{U}\left(\eta^{*}(\cdot), t\right) .
$$

\footnotetext{
${ }^{22}$ Since the conditional expectation above only depends on the process $\eta(\cdot)$ prior to date $t$, which is an arbitrary process anyway, we avoid complicating the notation unnecessarily in (65) by using the same letter to denote a candidate (possibly sub-optimal) level of effort in the infinitesimal interval $[t, t+d t]$.
} 
Hence, the process $e^{-r(.)} Z\left(\eta^{*}(\cdot), \cdot\right)$ is a square-integrable $\left\{\mathcal{F}_{t}\right\}$-martingale under the measure $\Pi_{c, \eta^{*}}^{E N}$. It follows from (57) and the martingale representation theorem (see Oksendal, 2003) that there exists a square-integrable, $\left\{\mathcal{F}_{t}\right\}$-adapted process $\omega(\cdot)$ such that ${ }^{23}$

$d Z\left(\eta^{*}(\cdot) ; t\right)-r Z\left(\eta^{*}(\cdot) ; t\right) d t=\omega(t) d W_{c, \eta^{*}}^{E N}(t)=\omega(t) s^{-1}\left[d \ln V(t)-\left(A c(t)^{\alpha} \eta^{*}(t)^{\beta}-\lambda s-l(t)\right) d t-\mu_{t}^{E N} d t\right]$.

Since the expectation in the dynamic programming equation (65) is taken under the measure $\Pi_{c, \eta}^{E N}$, it follows from $(57)$ and $(70)$ that $Z\left(\eta^{*}(\cdot) ; t\right)$ evolves under this measure as

$$
d Z\left(\eta^{*}(\cdot) ; t\right)=r Z\left(\eta^{*}(\cdot) ; t\right) d t+\omega(t) s^{-1} A c(t)^{\alpha}\left(\eta(t)^{\beta}-\eta^{*}(t)^{\beta}\right) d t+\omega(t) d W_{c, \eta}^{E N}(t) .
$$

Substituting (71) in (68) yields

$$
\begin{gathered}
d Z(\eta(\cdot) ; t)=r Z\left(\eta^{*}(\cdot) ; t\right) d t+\omega(t) s^{-1} A c(t)^{\alpha}\left(\eta(t)^{\beta}-\eta^{*}(t)^{\beta}\right)+k\left(\eta(t)^{\gamma}-\eta^{*}(t)^{\gamma}\right) V(t) d t \\
+r X(\eta(\cdot), t) d t+\omega(t) d W_{c, \eta}^{E N}(t) .
\end{gathered}
$$

Having derived the requisite expression for $d Z(\eta(\cdot) ; t)$, we substitute it in (65) to obtain

$$
\eta^{*}(t)=\operatorname{argmax}_{\eta(t)}\left[\omega(t) s^{-1} A c(t)^{\alpha} \eta(t)^{\beta}+k \eta(t)^{\gamma} V(t)\right]
$$

It then follows that the effort $\eta^{*}(t)$ is optimal over the interval $[t, t+d t]$ only if

$$
\frac{\omega(t)}{V(t)}=-\frac{k s}{A c(t)^{\alpha}} \frac{\gamma}{\beta}\left(\eta^{*}(t)\right)^{\frac{\gamma-\beta}{\beta}} .
$$

From the definition of the certainty equivalent process $R\left(\eta^{*}(\cdot) ; \cdot\right)$ in $(59)$, and using $(69)$, we have

$$
R\left(\eta^{*}(\cdot) ; t\right)=Z\left(\eta^{*}(\cdot) ; t\right)+\int_{0}^{t} e^{-r(u-t)} k \eta^{*}(u)^{\gamma} V(u) d u .
$$

Using (70)-(72) and (74), we obtain

$$
d R\left(\eta^{*}(\cdot) ; t\right)=a(t) V(t) d t+b(t) d V(t)
$$

where $b(t)$ and $a(t)$ are given by (63) and (64), respectively, as claimed.

Since the EN's maximum expected future utility from continuing the relationship at date $t \leq \tau$ is $\left.R\left(\eta^{*}(\cdot) ; t\right)\right)$ and since his utility from terminating the relationship is $P(t)$, the EN continues the relationship until time $\tau$ only if $P(t) \leq R\left(\eta^{*}(\cdot) ; t\right)$ at each date $t$. Replacing $t$ with the stopping time $\tau$ and $\eta(\cdot)$ with $\eta^{*}(\cdot)$ in $(58)$ and $(59)$, we see that $P(\tau)=R\left(\eta^{*}(\cdot) ; \tau\right)$. Hence, $P(t)=R\left(\eta^{*}(\cdot) ; t\right)$ for any time $t$ such that $\tau=t$ has positive probability. We conclude that $P(t)=R\left(\eta^{*}(\cdot) ; t\right)$ almost surely. Since the initial termination payoff equals the seed investment made by the VC, $P(0)=0$.

\footnotetext{
${ }^{23}$ Identity (70) is an almost sure relation that holds under all equivalent probability measures on the probability space. It is only under the measure $\Pi_{c, \eta^{*}}^{E N}$ defined in (55) that the process $\left[d V(t)-\left(A c(t)^{\alpha} \eta^{*}(t)^{\beta}-l(t)\right) d t-\mu_{t}^{E N} d t\right]$ is the increment of a Brownian motion.
} 
b) This result follows immediately from part a).

As a consequence of Lemma 1 , we can restrict consideration to contracts $P$ where

$$
d P(t)=a(t) V(t) d t+b(t) d V(t)
$$

where $b(t)>0$. Further, it follows from (63) that there is a one-one correspondence between the pay-performance sensitivity $b(t)$ and the EN's optimal effort $\eta(t)$ at date $t$.

\section{Optimal Contract Choice by VC}

By (63) and (64), a feasible contract is completely described by the investment process $c(\cdot)$, the processes $a(\cdot), b(\cdot)$ describing the fixed and proportional components of the EN's compensation and the termination time $\tau$. Define

$$
M_{a, b, c, \tau}(0)=E_{c, \eta}^{V C}\left[e^{-r \tau}(V(\tau)-P(\tau))-\int_{0}^{\tau} e^{-r s} c(s) V(s) d s\right]
$$

as the VC's discounted expected future payoff at date 0 if she chooses a contract $(P(\cdot), c(\cdot), \tau) \equiv$ $(a(\cdot), b(\cdot), c(\cdot), \tau)$. The VC's contract choice problem is then the following stochastic control problem

$$
\left(a^{*}(\cdot), b^{*}(\cdot), c^{*}(\cdot), \tau^{*}\right)=\operatorname{argmax}_{(a, b, c, \tau)} M_{a, b, c, \tau}(0) .
$$

Let the "state" of the system at any date $t$ be described by the ordered pair $\left(t, \mu_{t}^{V C}\right)$. We first restrict consideration to Markov controls where $a(t), b(t), c(t)$ and the decision to terminate

the relationship only depend on the current state $\left(t, \mu_{t}^{V C}\right)$. We derive the optimal Markov control policy. We then appeal to the verification theorem of dynamic programming (see Theorem 11.2.3 of Oksendal, 2003) to conclude that the optimal Markov control policy is, in fact, the optimal control policy over the entire space of admissible $\left\{\mathcal{F}_{t}\right\}$-adapted controls.

We note from (63) and (64) that the control $a(\cdot)$ is, in fact, determined by the controls $b(\cdot), c(\cdot)$ and the state of the system. Hence, a Markov control policy is completely described by $(b(\cdot), c(\cdot), \tau)$. For simplicity, we abuse notation by denoting the VC's continuation value in state $\left(t, \mu_{t}^{V C}\right)$ from adopting the Markov control policy $(b(\cdot), c(\cdot), \tau)$ by

$$
M_{b, c, \tau}\left(t, \mu_{t}^{V C}\right)=E_{t ; c, \eta}^{V C}\left[\left(e^{-r(\tau-t)} V(\tau)-V(t)\right)-\left(e^{-r(\tau-t)} P(\tau)-P(t)\right)-\int_{t}^{\tau} e^{-r(s-t)} c(s) V(s) d s\right]
$$

where $\eta(\cdot)$ is determined by inverting (63). Let $M^{*}\left(t, \mu_{t}^{V C}\right)$ be the optimal continuation value within the space of Markov controls and $\left(b^{*}(\cdot), c^{*}(\cdot), \tau^{*}\right)$ be the optimal Markov control policy. Suppose that the VC deviates from the optimal policy over the infinitesimal time interval $[t, t+d t]$ 
by choosing the controls $(\widehat{b}(t), \widehat{c}(t))$, Let $\widehat{M}\left(t, \mu_{t}^{V C}\right)$ denote the VC's continuation value at date $t$ under this deviated policy. It follows from (77) and (80) that

$$
\widehat{M}\left(t, \mu_{t}^{V C}\right)=E_{t ; c, \eta}^{V C}\left[-\widehat{a}(t) V(t) d t+(1-\widehat{b}(t)) d V(t)-\widehat{c}(t) V(t) d t+e^{-r d t} M^{*}\left(t+d t, \mu_{t+d t}^{V C}\right)\right] .
$$

By (56), we have

$$
\begin{aligned}
\widehat{M}\left(t, \mu_{t}^{V C}\right)=E_{t ; c, \eta}^{V C}[ & -\widehat{a}(t) V(t) d t+(1-\widehat{b}(t))\left[\mu_{t}^{V C}+A \widehat{c}(t)^{\alpha} \widehat{\eta}(t)^{\beta}-l(t)\right] V(t) d t-\widehat{c}(t) V(t) d t \\
& \left.+e^{-r d t} M^{*}\left(t+d t, \mu_{t+d t}^{V C}\right)\right]
\end{aligned}
$$

where $\widehat{\eta}(t)$ is given by (63) with $\widehat{b}(t)$ replacing $b(t)$. Since the VC's investment and EN's effort are

observable, the VC's assessment $\mu_{t+d t}^{V C}$ of project quality at date $t+d t$ is independent of the choices of controls $(\widehat{b}(t), \widehat{c}(t))$. Hence, the function $M^{*}\left(t+d t, \mu_{t+d t}^{V C}\right)$ is also independent of these choices. By the principle of optimality of dynamic programming, the optimal controls $\left(b^{*}(t), c^{*}(t)\right)$ at date $t$ must maximize the "flow" term in (82), that is,

$$
\left(b^{*}(t), c^{*}(t)\right)=\operatorname{argmax}_{\widehat{b}(t), \widehat{c}(t)}\left\{-\widehat{a}(t) d t+(1-\widehat{b}(t))\left[\mu_{t}^{V C}+A \widehat{c}(t)^{\alpha} \widehat{\eta}(t)^{\beta}-l(t)\right] d t-\widehat{c}(t) d t\right\} .
$$

We can now use the arguments described in detail in Section 3 to show that the optimal controls $\left(a^{*}(t), b^{*}(t), c^{*}(t)\right)$ are as described in Theorem 1. Further, the optimal termination time is the solution of the optimal stopping problem described in Section 5. The Markov control policy can be shown to satisfy the conditions of the dynamic programming verification theorem (see Section 11 of Oksendal, 2003). Hence, it is, in fact, the optimal control policy among the space of all square-integrable $\left\{\mathcal{F}_{t}\right\}$-adapted controls. This completes the proof of Theorem 1.

\section{Appendix C: Proofs of Remaining Results}

The incremental change in termination payoff (1) depends on $\eta$ only through the terms $\eta^{\beta}, \eta^{\gamma}$. There is no loss of generality if the unit of effort is redefined as $z:=\eta^{\beta}$, the production function is taken as $c^{\alpha} z$ and the disutility of effort is taken as $z^{\gamma / \beta}$. As characterized in Theorem 1 , the equilibrium depends on the parameters $\beta$ and $\gamma$ only through their ratio $\gamma / \beta$. To simplify the notation in the proofs to follow, we shall hereafter normalize $\beta$ to 1.

For each parameter " $\Pi$ " (e.g. $\sigma_{0}^{2}, s^{2}, \lambda, \Delta_{0}, k$ ) we let $b_{t}(\pi)$ denote the solution to (37) at time $t$, define $c_{t}(\pi):=c\left(b_{t}(\pi)\right)$, and let $b(\pi)$ and $c(\pi)$ denote the corresponding time paths when the parameter ח's value equals $\pi$. We shall write $F_{t}^{\prime}(b, \pi)$ when we wish to make explicit the functional dependence of the derivative of $F_{t}$ on the parameter value $\pi$. For subsequent reference, 
the derivative of the VC's Periodic Flow Function (36) is given by

$$
F_{t}^{\prime}(b)=\Delta_{t}-p+K c^{\prime}(b)=\frac{s^{2}}{s^{2}+t \sigma_{0}^{2}} \Delta_{0}-\lambda s+K c^{\prime}(b) .
$$

The following Lemma will be used repeatedly in the proofs to follow.

\section{Lemma 2}

If $F_{t}^{\prime}(b, \pi)$ is an increasing (decreasing) function of $\pi$, then $b_{t}(\pi)$ is an increasing (decreasing) function of $\pi$.

Proof. Let $\pi^{1}<\pi^{2}$. Suppose first that $F_{t}^{\prime}(b, \pi)$ is an increasing function of $\pi$. By definition,

$$
0=F_{t}^{\prime}\left(b_{t}\left(\pi^{2}\right), \pi^{2}\right)=F_{t}^{\prime}\left(b_{t}\left(\pi^{1}\right), \pi^{1}\right)<F_{t}^{\prime}\left(b_{t}\left(\pi^{1}\right), \pi^{2}\right),
$$

which immediately implies $b_{t}\left(\pi^{1}\right)<b_{t}\left(\pi^{2}\right)$ by the strict concavity of $F_{t}$. The proof in the decreasing case is analogous.

Proof of Proposition 1. The optimal investment function is clearly positive on $(0,1]$. The marginal optimal investment is given by

$$
c^{\prime}(b) \propto\left(\frac{1}{k}\right)^{\frac{1}{(1-\alpha) \gamma-1}} b^{r_{1}}(\gamma-b)^{r_{2}}(1-b)
$$

where $r_{1}:=\frac{2-(1-\alpha) \gamma}{(1-\alpha) \gamma-1}$ and $r_{2}:=\frac{\alpha \gamma}{(1-\alpha) \gamma-1}$ and where the symbol $\propto$ means "equal up to a positive multiplicative constant". Under Assumption 2, the parameter $r_{2}$ is positive and the parameter $r_{1}$ is negative. (Keep in mind that $\beta$ is now 1.) Since $\gamma>1$ (Assumption 1), it follows that $c^{\prime}(\cdot)>0$ on $[0,1), c^{\prime}(1)=0$ and $c^{\prime}(\cdot)$ is negative on $[1, \gamma / \beta)$. This establishes that $c(\cdot)$ is increasing on $[0,1]$ and achieves it maximum value at $b=1$. The second derivative is given by

$$
c^{\prime \prime}(b) \propto b^{r_{1}-1}(\gamma-b)^{r_{2}-1}\left[r_{1}(\gamma-b)(1-b)-r_{2} b(1-b)-b(\gamma-b)\right] .
$$

The function $c(\cdot)$ is strictly concave on $(0,1]$ since $c^{\prime \prime}(\cdot)$ is negative on $(0,1]$. Part (a) has been established. Given that $\Delta_{t}$ decreases with $t$, it follows directly from Assumption 2 and part (a) that the solution to $(37)$ will belong to $(0,1)$. On this interval, the VC's Periodic Flow $F_{t}(\cdot)$ is strictly concave by part (a), and hence possesses a unique maximum. This establishes part (b).

Proof of Theorem 2. It follows directly from (84) and Lemma 2 that the EN's pay performance sensitivity decreases with time where here the parameter $\Pi=t$. This in turn implies the investment rates decrease with time by Proposition 1. Since both the pay performance sensitivities and investment rates decrease with time, the effort levels in (23) decrease with time, too.

Proof of Theorem 3. We begin with parts (a)-(c). By Proposition (1) and the form for the optimal effort level (23), the results for the optimal investment rate and EN's effort paths will 
follow if we show that the path of the EN's pay performance sensitivity is a pointwise decreasing function of the respective parameters. This follows directly from (84) and Lemma 2 for either $\sigma_{0}^{2}$ or $\lambda$. We turn our attention to $s^{2}$. Fix $s$ such that $\Delta_{0}<\lambda s$. We have

$$
\frac{\partial F_{t}^{\prime}\left(b_{t}(s), s\right)}{\partial s}=\frac{2 s \Delta_{0} t \sigma_{0}^{2}}{\left(s^{2}+t \sigma_{0}^{2}\right)^{2}}-\lambda<\lambda\left[\frac{2 t \sigma_{0}^{2} s^{2}}{\left(s^{2}+t \sigma_{0}^{2}\right)^{2}}-1\right]=-\lambda\left[\frac{\left(s^{4}+\left(t \sigma_{0}^{2}\right)^{2}\right.}{\left(s^{2}+t \sigma_{0}^{2}\right)^{2}}\right]<0 .
$$

Consequently, $\partial F_{t}^{\prime}\left(b_{t}(s), s\right) / \partial s<0$, which establishes the result for the intrinsic risk by direct application of Lemma 2. The proof of parts (a)-(c) is complete. Part (d) follows directly from (84) and Lemma 2 and the same arguments given in the proof of parts (a)-(c). As for part (e), an examination of $(85)$ shows that if $c^{\prime}(b)>0$, then $c^{\prime}(b)$ decreases with $k$. Since $c^{\prime}(\cdot)$ is positive on $[0,1)$, the result now follows directly from (84) and Lemma 2 and previous arguments.

\section{Proof of Proposition 2. Let}

$$
\phi\left(t, \mu_{t}, \tau\right):=E_{t}^{V C}\left[\int_{t}^{\tau}\left(F_{v}^{*}-\ell_{v}+\Theta-R_{b}\right) d v\right]
$$

denote the VC's continuation value function at date $t$ given her current project assessment $\mu_{t}$ and a given (possibly sub-optimal) stopping time $\tau$. In the above, $F_{v}^{*}$ satisfies (37). The VC's optimal continuation value function is

$$
\phi^{*}\left(t, \mu_{t}\right):=\sup _{\tau \geq t} \phi\left(t, \mu_{t}, \tau\right)
$$

By standard dynamic programming arguments, the optimal termination time $\tau^{*}$ (if it exists) must solve (87) for any $t \in[0, T]$ and $\mu_{t} \in(-\infty, \infty)$. Further, the VC continues the project at any date $t$ and project assessment $\mu_{t}$ if and only if $\phi^{*}\left(t, \mu_{t}\right)>0$. The proof proceeds by showing that $\phi^{*}(t, \cdot)$ is monotonic (non-decreasing) and lower semi-continuous. It then follows that at each date $t \in[0, T]$ there exists a trigger $\mu_{t}^{*}$ such that the VC continues the project if and only if $\mu_{t}>\mu_{t}^{*}$.

We prove the monotonicity and lower semi-continuity of $\phi^{*}(t, \cdot)$ by considering the sequence of discrete stopping time problems in which for each fixed positive integer $N$ the $\mathrm{VC}$ is constrained to terminate the project only at the discrete set of times $\left\{0, \frac{T}{2^{N}}, \ldots, \frac{\left(2^{N}-1\right) T}{2^{N}}, T\right\}$. We show that the VC's optimal value functions $\phi_{N}^{*}(t, \cdot)$ in the discrete problems are continuous and monotonic. We then use a convergence argument to show that $\phi^{*}(t, \cdot)$ is lower semi-continuous and monotonic.

Pick a positive integer $N$. We use backward induction to show continuity and monotonicity of $\phi_{N}^{*}(t, \cdot)$. To establish continuity we further show there exists positive constants $\kappa_{t}^{1}, \kappa_{t}^{2}$ such that

$$
\phi_{N}^{*}\left(t, \mu_{t}\right) \leq \kappa_{t}^{1}+\kappa_{t}^{2} \max \left\{\mu_{t}, 0\right\}
$$

The assertions of continuity, monotonicity and (88) are trivial at date $T$ since $\phi_{N}^{*}(T, \cdot) \equiv 0$. Suppose that the assertion is true for $t \in\left[t^{\prime}+\frac{1}{2^{N}}, \ldots, T\right]$. We will establish that the assertion is true for 
$t \in\left[t^{\prime}, t^{\prime}+\frac{1}{2^{N}}\right)$. Consider first any $t \in\left(t^{\prime}, t^{\prime}+\frac{1}{2^{N}}\right]$. By the dynamic programming principle,

$$
\phi_{N}^{*}\left(t, \mu_{t}\right)=E_{t}\left[\int_{t}^{t^{\prime}+\frac{1}{2^{N}}}\left(F_{v}^{*}-\ell_{v}+\Theta-R_{b}\right) d v+\phi_{N}^{*}\left(t^{\prime}+\frac{1}{2^{N}}, \mu_{t^{\prime}+\frac{1}{2^{N}}}\right)\right] .
$$

Since $F_{v}^{*}$ is bounded and deterministic by (37), (7), (29) and (30) and $\Theta$ is normally distributed,

$$
E_{0}^{V C}\left[\int_{0}^{T}\left(\left(F_{v}^{*}\right)^{2}+\left(\ell_{v}\right)^{2}+(\Theta)^{2}+\left(R_{b}\right)^{2}\right) d v\right]<\infty
$$

We can therefore apply Fubini's theorem to conclude that

$$
\phi_{N}^{*}\left(t, \mu_{t}\right)=\left[\int_{t}^{t^{\prime}+\frac{1}{2^{N}}}\left(F_{v}^{*}-\ell_{v}+\mu_{t}-R_{b}\right) d v+E_{t} \phi_{N}^{*}\left(t^{\prime}+\frac{1}{2^{N}}, \mu_{t^{\prime}+\frac{1}{2^{N}}}\right)\right] .
$$

We first establish monotonicity of $\phi_{N}^{*}(t, \cdot)$. The integral on the right-hand side of (91) obviously increases with $\mu_{t}$; it remains to show the expectation on the right-hand side of (91) is also monotonic in $\mu_{t}$. A bit of algebra applied to (2) and (4) shows that $\mu_{t^{\prime}+\frac{1}{2^{N}}} \sim N\left(\mu_{t}, \hat{\sigma}^{2}\right)$ is normally distributed. Further, $\mu_{t^{\prime}+\frac{1}{2^{N}}}$ may be expressed in the form $f_{t}\left(\mu_{t}, Z\right)$ where $Z \sim N(0,1)$ and $f_{t}(\cdot, \cdot)$ is an increasing, linear function of its arguments. The monotonicity of $E_{t} \phi_{N}^{*}\left(t^{\prime}+\frac{1}{2^{N}}, \cdot\right)$ now follows from

$$
E_{t} \phi_{N}^{*}\left(t^{\prime}+\frac{1}{2^{N}}, \mu_{t^{\prime}+\frac{1}{2^{N}}}\right)=E \phi_{N}^{*}\left(t^{\prime}+\frac{1}{2^{N}}, f_{t}\left(\mu_{t}, Z\right)\right),
$$

since the expectation on the right-hand side of (92) is taken with respect to the standard normal density, which is independent of the problem parameters, and since both $f_{t}(\cdot, z)$ and $\phi_{N}^{*}\left(t^{\prime}+\frac{1}{2^{N}}, \cdot\right)$ are monotonic in $\mu_{t}$ (the latter by the inductive assumption).

Next we show continuity of $\phi_{N}^{*}(t, \cdot)$. Once again, this property obviously holds for the integral on the right-hand side of (91); it remains to show the expectation on the right-hand side of (91) is also continuous in $\mu_{t}$. This result will follow from identity (92) if the limit and expectation operators may be interchanged, since both $f_{t}(\cdot, z)$ and $\phi_{N}^{*}\left(t^{\prime}+\frac{1}{2^{N}}, \cdot\right)$ are continuous in $\mu_{t}$ (the latter by the inductive assumption). By the inductive assumption (88) the function $\phi_{N}^{*}\left(t^{\prime}+\frac{1}{2^{N}}, \cdot\right)$ is bounded above by a positive function whose expectation

$$
E\left[\kappa_{t^{\prime}+\frac{1}{2^{N}}}^{1}+\kappa_{t^{\prime}+\frac{1}{2^{N}}}^{2} \max \left\{\mu_{t^{\prime}+\frac{1}{2^{N}}}, 0\right\}\right]=\kappa_{t^{\prime}+\frac{1}{2^{N}}}^{1}+\kappa_{t^{\prime}+\frac{1}{2^{N}}}^{2}\left\{\frac{\hat{\sigma}_{t}}{\sqrt{2 \pi}} e^{-1 / 2\left(\frac{\mu_{t}}{\tilde{\sigma}_{t}}\right)^{2}}+\mu_{t} P\left(Z>-\frac{\mu_{t}}{\hat{\sigma}_{t}}\right)\right\}
$$

is finite, and thus the interchange is justified by the dominated convergence theorem.

To complete the inductive argument we must show that (88) holds for $t$. The integral on the right-hand side of (91) is bounded above by $\left(t^{\prime}+\frac{1}{2^{N}}-t\right)\left(F_{0}^{*}+\max \left(\mu_{t}, 0\right)\right)$-recall the $F_{t}^{*}$ decrease with $t$. Since (88) holds for $t=t^{\prime}+\frac{1}{2^{N}}$, the inequality (93) shows that the expectation on the righthand side of (91) is bounded above by $\left(\kappa_{t^{\prime}+\frac{1}{2^{N}}}^{1}+\kappa_{t^{\prime}+\frac{1}{2^{N}}}^{2} \frac{\hat{\sigma}_{t}}{\sqrt{2 \pi}}\right)+\kappa_{t^{\prime}+\frac{1}{2^{N}}}^{2} \max \left(\mu_{t}, 0\right)$. It is therefore possible to define positive constants $\kappa_{t}^{1}, \kappa_{t}^{2}$ such that (88) holds for $t$, as required. 
Finally, we must establish the inductive step for $t=t^{\prime}$. We have

$$
\phi_{N}^{*}\left(t, \mu_{t}\right)=\max \left[0,\left[\int_{t^{\prime}}^{t^{\prime}+\frac{1}{2^{N}}}\left(F_{v}^{*}-\ell_{v}+\mu_{t^{\prime}}-R_{b}\right) d v+E_{t^{\prime}} \phi_{N}^{*}\left(t^{\prime}+\frac{1}{2^{N}}, \mu_{t^{\prime}+\frac{1}{2^{N}}}\right)\right]\right],
$$

where (94) differs from (91) because the VC can terminate at date $t^{\prime}$. It should be clear that the previous arguments still apply, and hence the inductive step is established.

Because $\left\{0, \frac{1}{2^{N}}, \ldots, \frac{\left(2^{N}-1\right) T}{2^{N}}, T\right\} \subset\left\{0, \frac{1}{2^{N^{\prime}}}, \ldots, \frac{\left(2^{N^{\prime}}-1\right) T}{2^{N^{\prime}}}, T\right\}$ for all $N<N^{\prime}$, it follows that $\phi_{N}^{*}\left(t, \mu_{t}\right) \leq \phi_{N^{\prime}}^{*}\left(t, \mu_{t}\right)$. For each $\left(t, \mu_{t}\right) \in[0, T] \times(-\infty, \infty)$ we may therefore define

$$
\bar{\phi}\left(t, \mu_{t}\right):=\lim _{N \rightarrow \infty} \phi_{N}^{*}\left(t, \mu_{t}\right) .
$$

We claim that $\bar{\phi}=\phi^{*}$. Fix $\left(t, \mu_{t}\right) \in[0, T] \times(-\infty, \infty)$. Since $\phi^{*}\left(t, \mu_{t}\right) \geq \phi_{N}^{*}\left(t, \mu_{t}\right)$ for all $N$, $\phi^{*}\left(t, \mu_{t}\right) \geq \bar{\phi}\left(t, \mu_{t}\right)$. Suppose that $\phi^{*}\left(t, \mu_{t}\right)>\bar{\phi}\left(t, \mu_{t}\right)$. Choose any $\epsilon<\left(\phi^{*}\left(t, \mu_{t}\right)-\bar{\phi}\left(t, \mu_{t}\right)\right) / 2$. There exists a stopping time $\tau^{\epsilon}$ such that $\bar{\phi}\left(t, \mu_{t}\right)<\phi^{*}\left(t, \mu_{t}\right)-\epsilon<\phi\left(t, \mu_{t}, \tau^{\epsilon}\right)$ where $\phi\left(t, \mu_{t}, \tau^{\epsilon}\right)$ is defined in (86). Define the stopping time $\tau_{N}^{\epsilon}=\frac{i}{2^{N}} 1_{\left\{\frac{i}{2^{N}}<\tau^{\epsilon}<\frac{i+1}{2^{N}}\right\}}$. There exists $N$ sufficiently large such that $\phi\left(t, \mu_{t}, \tau_{N}^{\epsilon}\right)>\phi\left(t, \mu_{t}, \tau^{\epsilon}\right)-\epsilon$. It follows that $\bar{\phi}\left(t, \mu_{t}\right)<\phi\left(t, \mu_{t}, \tau_{N}^{\epsilon}\right)$. By definition of the function $\phi_{N}^{*}\left(t, \mu_{t}\right)$, however, $\phi\left(t, \mu_{t}, \tau_{N}^{\epsilon}\right) \leq \phi_{N}^{*}\left(t, \mu_{t}\right) \leq \bar{\phi}\left(t, \mu_{t}\right)$, which is a contradiction. Hence, $\bar{\phi}\left(t, \mu_{t}\right)=\phi^{*}\left(t, \mu_{t}\right)$. The monotonicity of $\phi^{*}(t, \cdot)$ easily follows from the monotonicity of $\phi_{N}^{*}(t, \cdot)$ and the fact that $\phi^{*}\left(t, \mu_{t}\right)=\lim _{N \rightarrow \infty} \phi_{N}^{*}\left(t, \mu_{t}\right)$. The lower semi-continuity of $\phi^{*}(t, \cdot)$ follows from the fact that the supremum of continuous functions is lower semi-continuous.

Proof of Proposition 3. The function $F_{t}(\cdot)$ in $(36)$ is an increasing function of $\Delta_{0}$, which implies that $F_{t}^{*}$ is also a increasing function of $\Delta_{0}$. Consequently, the function $\phi_{t}^{*}$ in (87) increases with $\Delta_{0}$, too. Since a change in $\Delta_{0}$ has no effect on the sample paths, and the path of $\mu_{t}^{V C}$ is continuous almost surely, the project duration increases with $\Delta_{0}$. Since $F_{t}(\cdot)$ decreases function with $\lambda$ or $k$, analogous arguments show that the project duration also decreases with $\lambda$ and $k$.

Proof of Proposition 4. Pick $\epsilon>0$ and define $\theta_{0}$ so that $P\left(\Theta>\theta_{0}\right)=\epsilon$. Now

$$
\begin{aligned}
P(\tau>T) & =P\left\{\mu_{t}>\mu_{t}^{*} \text { for all } t \in[0, T]\right\} \\
& \leq P\left\{\mu_{T}>\mu_{T}^{*} \mid \Theta \leq \theta_{0}\right\} P\left(\Theta \leq \theta_{0}\right)+P\left(\Theta>\theta_{0}\right) \\
& \leq P\left\{\mu_{T}>\mu_{T}^{*} \mid \Theta=\theta_{0}\right\}+\epsilon
\end{aligned}
$$

By Proposition 2 and the assumed property of the $l_{t}$, the $\mu_{t}^{*}$ eventually always lie above a positive constant. Further, because, the conditional distribution of $\int_{0}^{t} \xi_{u} d u / t$ given $\Theta=\theta_{0}$ is $N\left(\theta_{0}, s^{2} / t\right)$,

$$
\begin{aligned}
P\left\{\mu_{t} \geq \mu_{t}^{*} \mid \Theta=\theta_{0}\right\} & =P\left\{\frac{s^{2} \mu_{0}+\sigma_{0}^{2}\left(\int_{0}^{t} \xi_{u} d u\right)}{s^{2}+t \sigma_{0}^{2}} \geq \mu_{t}^{*} \mid \Theta=\theta_{0}\right\} \\
& \leq P\left\{\frac{\int_{0}^{t} \xi_{u} d u}{t} \geq \mu_{t}^{*}+\frac{s^{2}\left(\mu_{t}^{*}-\mu_{0}\right)}{t \sigma_{0}^{2}} \mid \Theta=\theta_{0}\right\} \rightarrow 0 \text { as } t \rightarrow \infty .
\end{aligned}
$$

The result now follows from (96) and (97) since $\epsilon$ was chosen arbitrarily. 
Table 1: Baseline Parameter Values (standard errors in parentheses) and Baseline Outputs

\begin{tabular}{|cccc||cccc||ccc|}
\hline \multicolumn{3}{|c||}{ Technology parameters } & \multicolumn{3}{c||}{ Belief parameters } & \multicolumn{3}{c|}{ Preference parameters } \\
\hline$A$ & $\alpha$ & $\ell_{1}$ & $\ell_{2}$ & $\mu_{0}^{V C}$ & $\Delta_{0}$ & $s$ & $\sigma_{0}$ & $\lambda$ & $k$ & $\gamma$ \\
0.8435 & 0.1631 & 0.0820 & 1.9638 & 0.0660 & 0.2457 & 0.7280 & 0.4840 & 0.5469 & 0.0220 & 5.8107 \\
$(0.038)$ & $(0.024)$ & $(0.004)$ & $(0.093)$ & $(0.002)$ & $(0.009)$ & $(0.015)$ & $(0.019)$ & $(0.021)$ & $(0.001)$ & $(0.255)$ \\
\hline
\end{tabular}

\begin{tabular}{|c|c|c|c|c|c|c|c|c|}
\hline \multirow[b]{2}{*}{ Agency Scenario } & \multirow[b]{2}{*}{ Project Value } & \multirow[b]{2}{*}{ VC Fund Stake } & \multirow[b]{2}{*}{ Investment } & \multicolumn{5}{|c|}{ Distribution of Project Duration } \\
\hline & & & & $p_{0}^{*}$ & $p_{1}^{*}$ & $p_{2}^{*}$ & $p_{3}^{*}$ & $E[\tau]$ \\
\hline Actual & 4.23 & 3.24 & 0.48 & 0.506 & 0.383 & 0.106 & 0.004 & 1.61 \\
\hline Symmetric & 3.47 & 2.91 & 0.38 & 0.565 & 0.341 & 0.090 & 0.003 & 1.53 \\
\hline No Agency & 7.08 & 2.67 & 0.87 & 0.327 & 0.467 & 0.192 & 0.014 & 1.89 \\
\hline
\end{tabular}

Table 2: Predicted and Observed Statistics

The first rows of the two tables record the observed statistics in Sahlman (1990, Figure 1) and Cochrane (2005, Table 4). The second rows record the model's predictions.

\begin{tabular}{|ccccc||ccccc||c||c|}
\hline \multicolumn{4}{|c||}{$\begin{array}{c}\text { Distribution } \\
\text { of Value }\end{array}$} & \multicolumn{4}{c||c}{$\begin{array}{c}\text { Distribution } \\
\text { of Investment }\end{array}$} & Investment \\
\hline$\underline{1}$ & $\underline{2}$ & $\underline{3}$ & $\underline{4}$ & $\underline{5}$ & $\underline{1}$ & $\underline{2}$ & $\underline{3}$ & $\underline{4}$ & $\underline{5}$ & \\
0.020 & 0.105 & 0.130 & 0.255 & 0.490 & 0.345 & 0.300 & 0.198 & 0.089 & 0.068 & 4.28 \\
0.045 & 0.117 & 0.176 & 0.209 & 0.454 & 0.319 & 0.292 & 0.180 & 0.110 & 0.099 & 4.32 \\
\hline
\end{tabular}

\begin{tabular}{|c|c|c|c|c|c|c|c|}
\hline \multicolumn{4}{|c|}{$\begin{array}{c}\text { Round-to-Round } \\
\text { Returns }\end{array}$} & \multicolumn{4}{|c|}{$\begin{array}{c}\text { Round-to-Round } \\
\text { Variances }\end{array}$} \\
\hline$\underline{1}$ & $\underline{2}$ & $\underline{3}$ & $\underline{4}$ & $\underline{1}$ & $\underline{2}$ & $\underline{3}$ & $\underline{4}$ \\
\hline 0.26 & 0.20 & 0.15 & 0.09 & 0.90 & 0.83 & 0.77 & 0.84 \\
\hline 0.25 & 0.20 & 0.16 & 0.10 & 0.88 & 0.85 & 0.81 & 0.83 \\
\hline
\end{tabular}

Table 3: Contract Parameter Values (first four periods)

\begin{tabular}{|c|c|c|c|c|c|c|c|c|}
\hline Agency Scenario & $b_{0}^{*}$ & $b_{1}^{*}$ & $b_{2}^{*}$ & $b_{3}^{*}$ & $c_{0}^{*}$ & $c_{1}^{*}$ & $c_{2}^{*}$ & $c_{3}^{*}$ \\
\hline Actual & 0.415 & 0.309 & 0.271 & 0.251 & 0.115 & 0.110 & 0.108 & 0.107 \\
Symmetric & 0.188 & 0.188 & 0.188 & 0.188 & 0.102 & 0.102 & 0.102 & 0.102 \\
No Agency & 1.000 & 1.000 & 1.000 & 1.000 & 0.123 & 0.123 & 0.123 & 0.123 \\
\hline
\end{tabular}




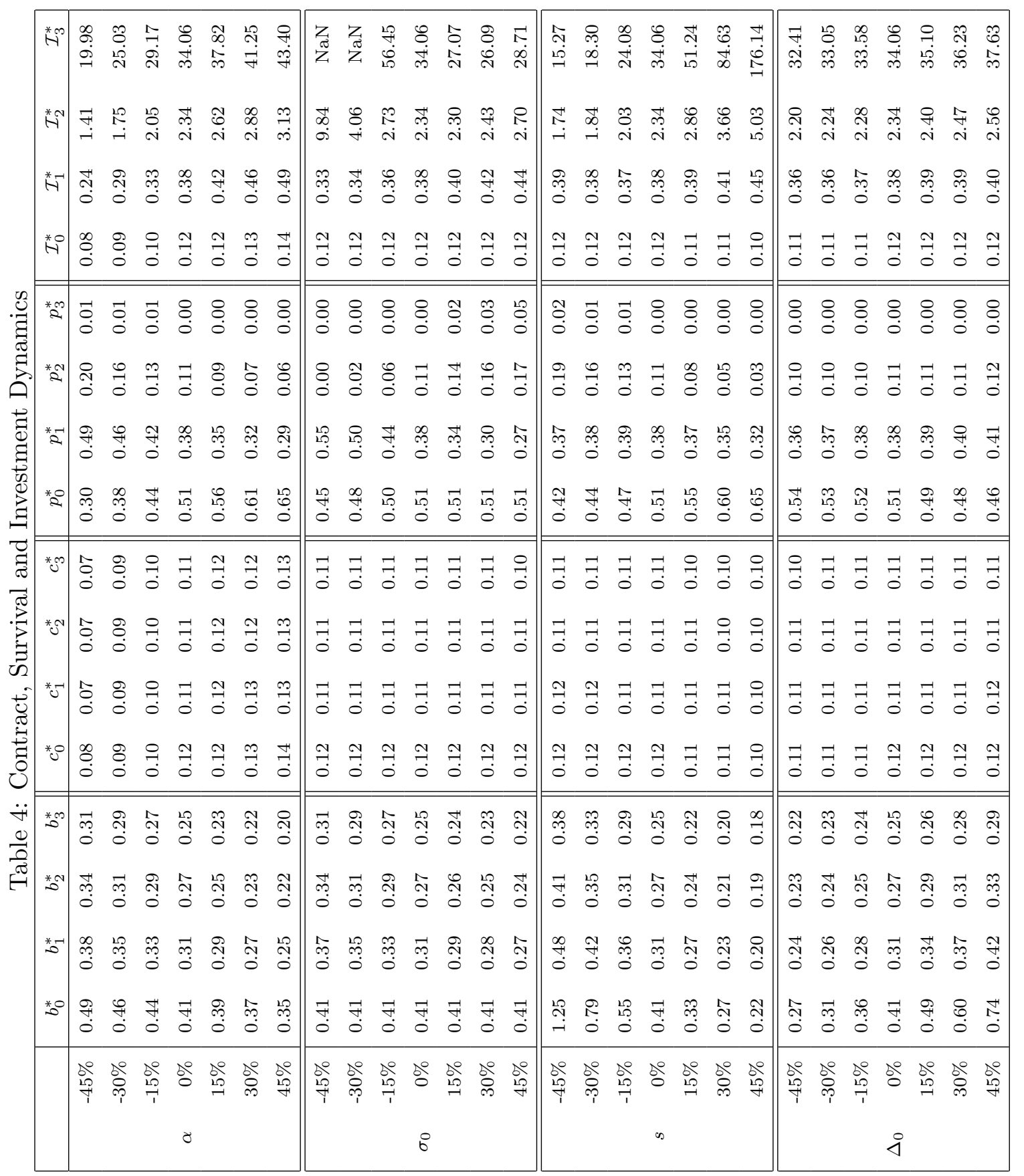


Figure 1: Project Value, VC Fund Stake, Investment (Left Hand Axis) and Expected Project Duration (Right Hand Axis) with Capital Intensity

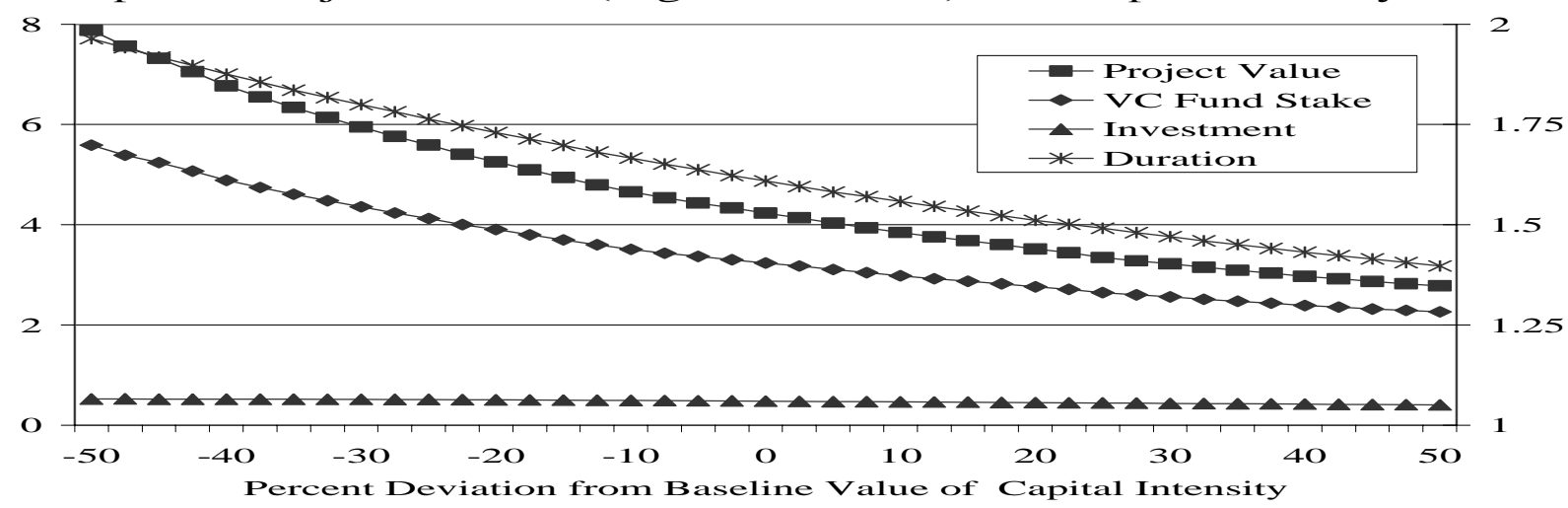

Figure 2: Project Value, VC Fund Stake, Investment (Left Hand Axis) and Expected Project Duration (Right Hand Axis) with Transient Risk

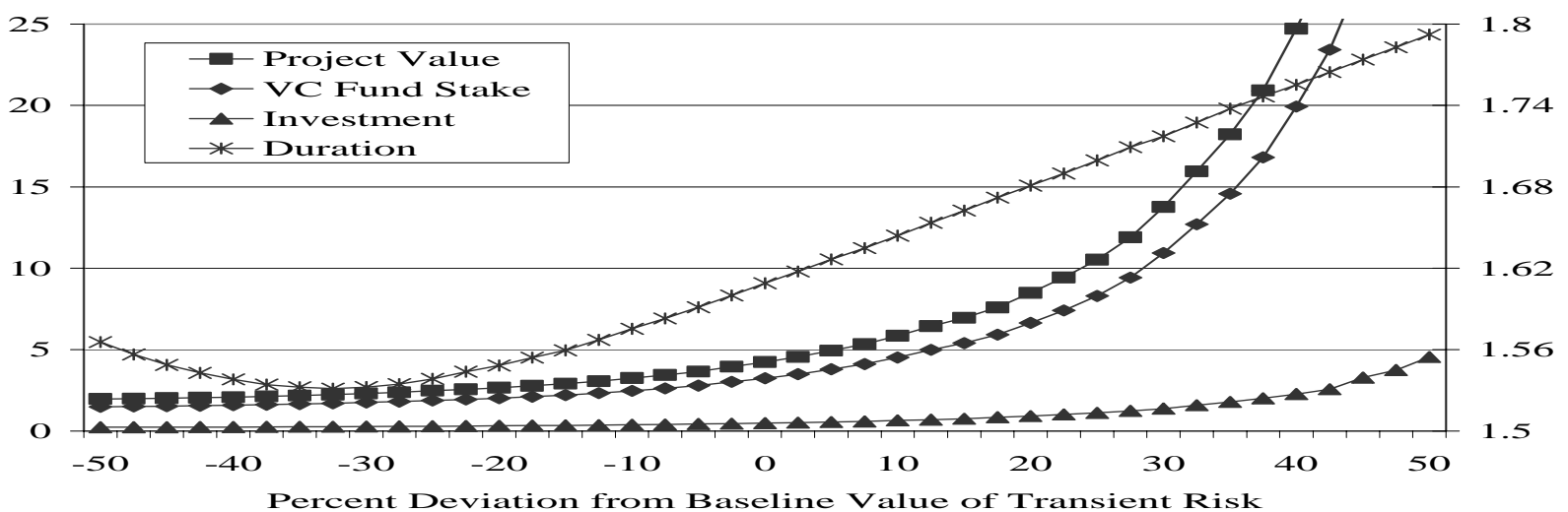

Figure 3: Project Value, VC Fund Stake, Investment (Left Hand Axis) and Expected Project Duration (Right Hand Axis) with Intrinsic Risk

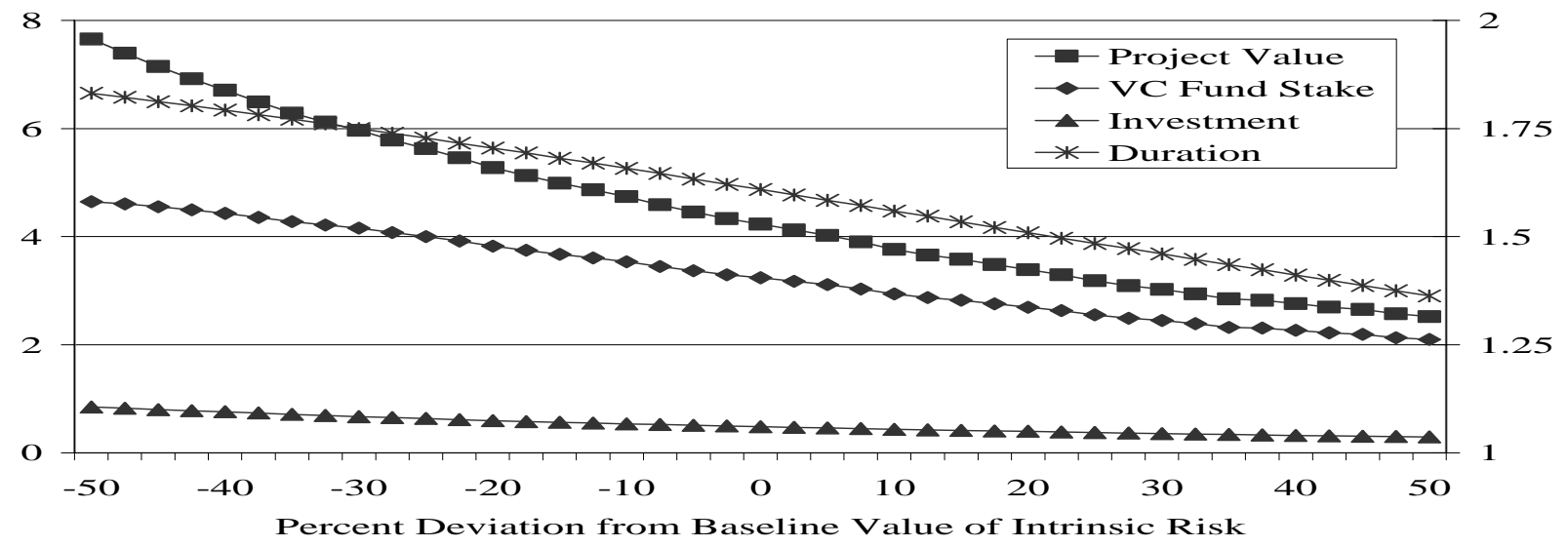

Figure 4: Project Value, VC Fund Stake, Investment (Left Hand Axis) and Expected Project Duration (Right Hand Axis) with Optimism

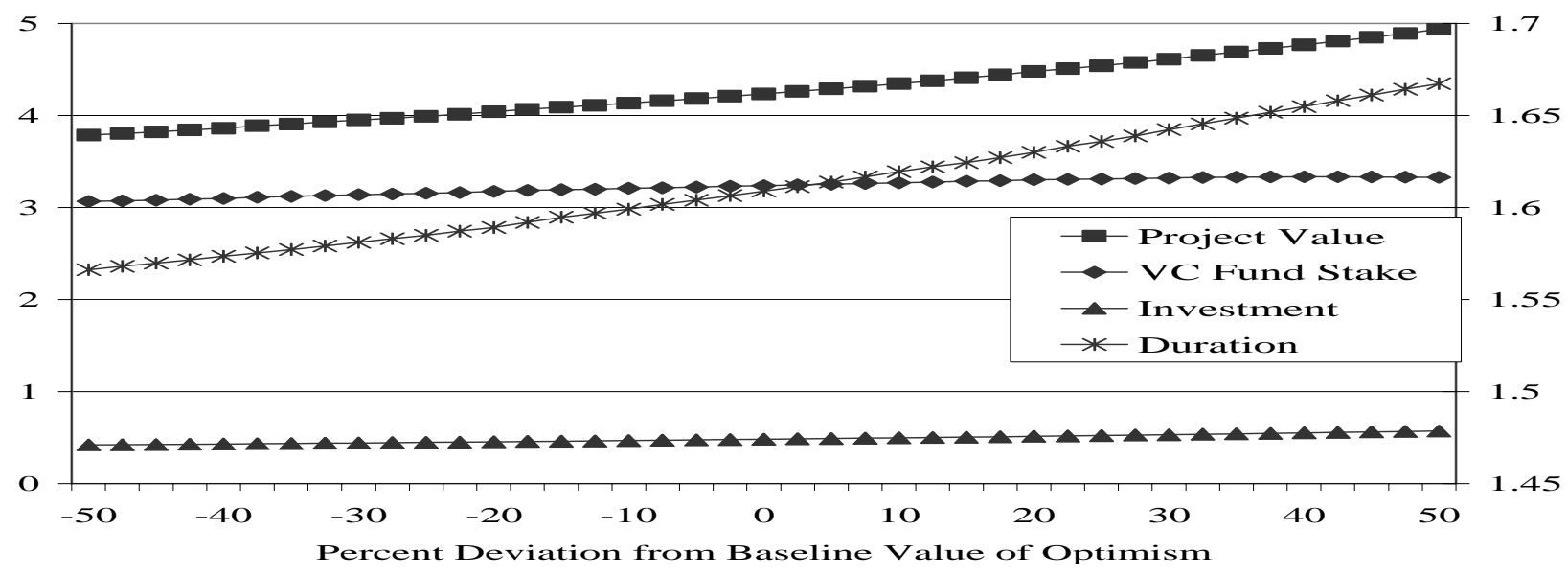


Figure 5: Implied Discount Rate with Capital Intensity, Transient Risk, Intrinsic Risk and Optimism

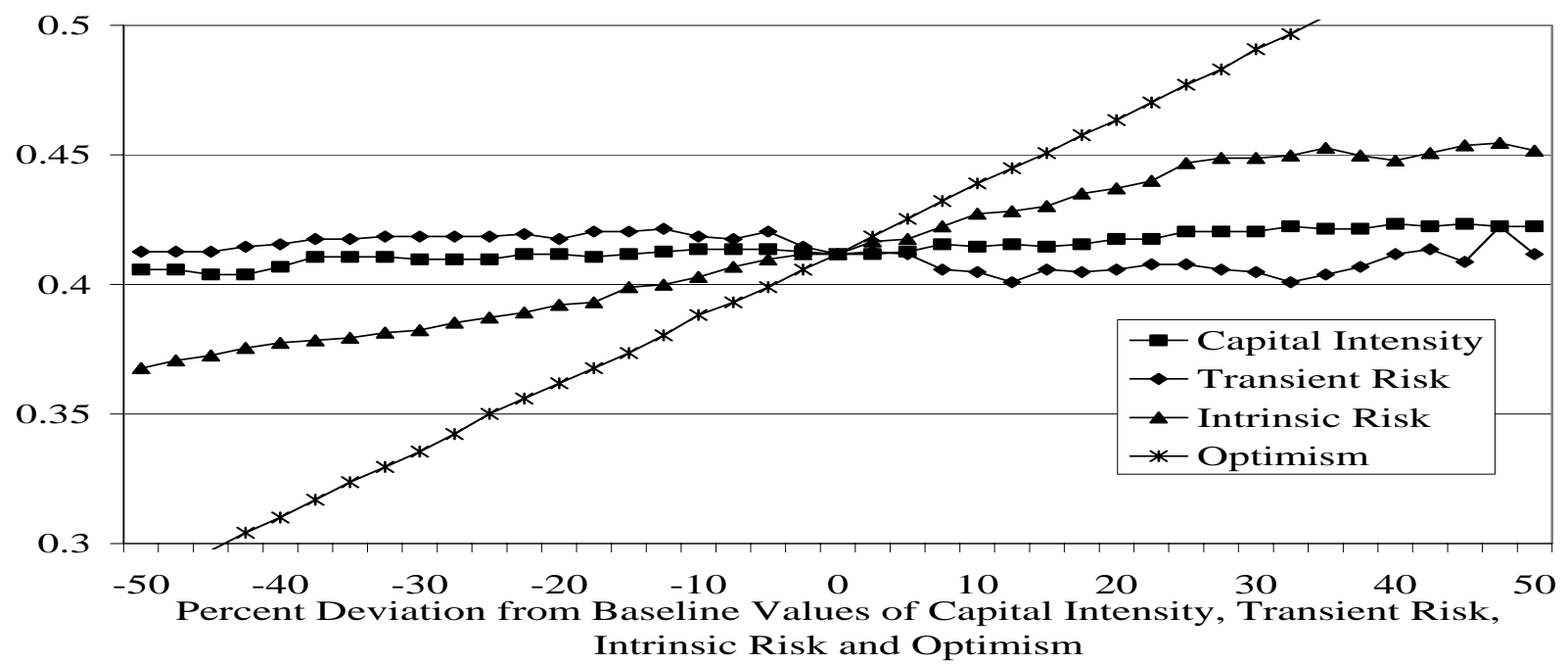

Figure 6: Optimism Ratio with Capital Intensity, Transient Risk, Intrinsic Risk and Optimism

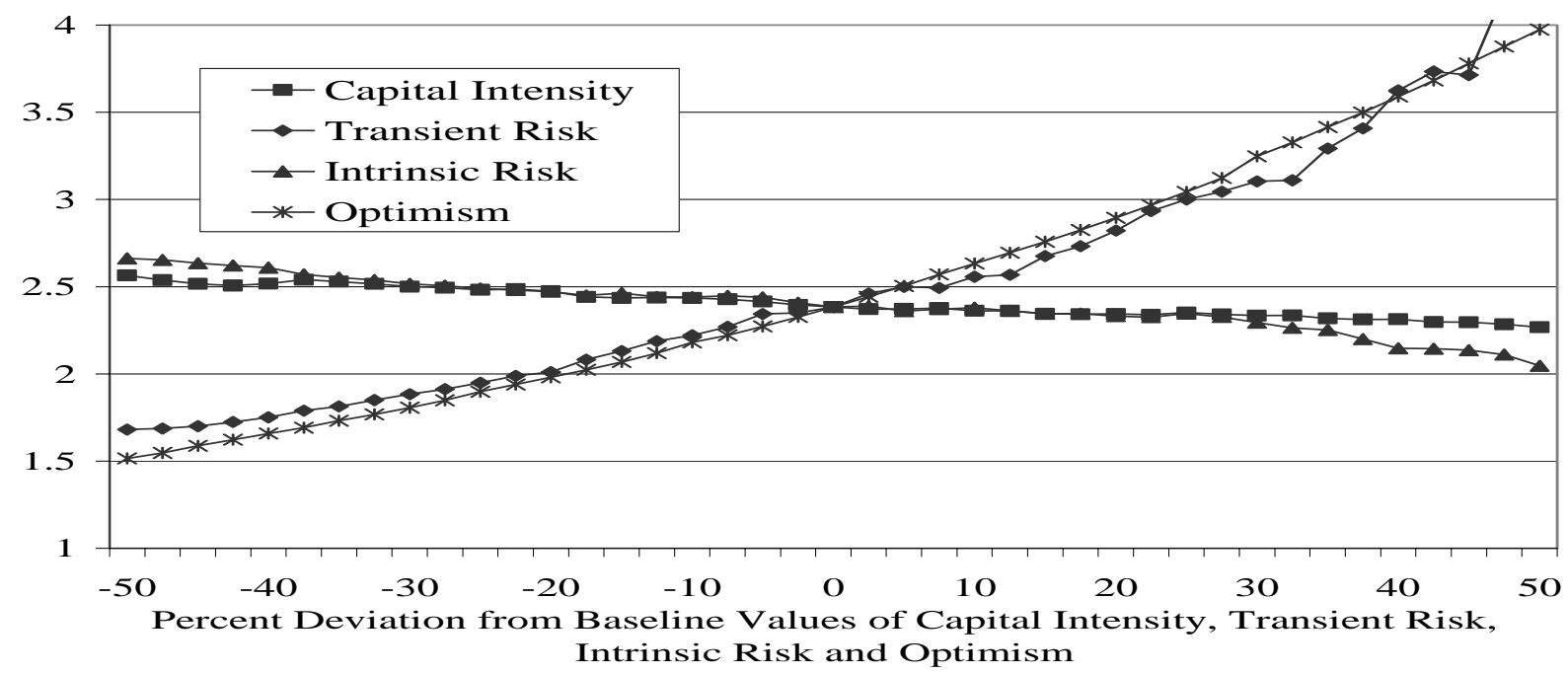

Figure 7: Internal Rate of Return with Capital Intensity, Transient Risk, Intrinsic Risk and Optimism

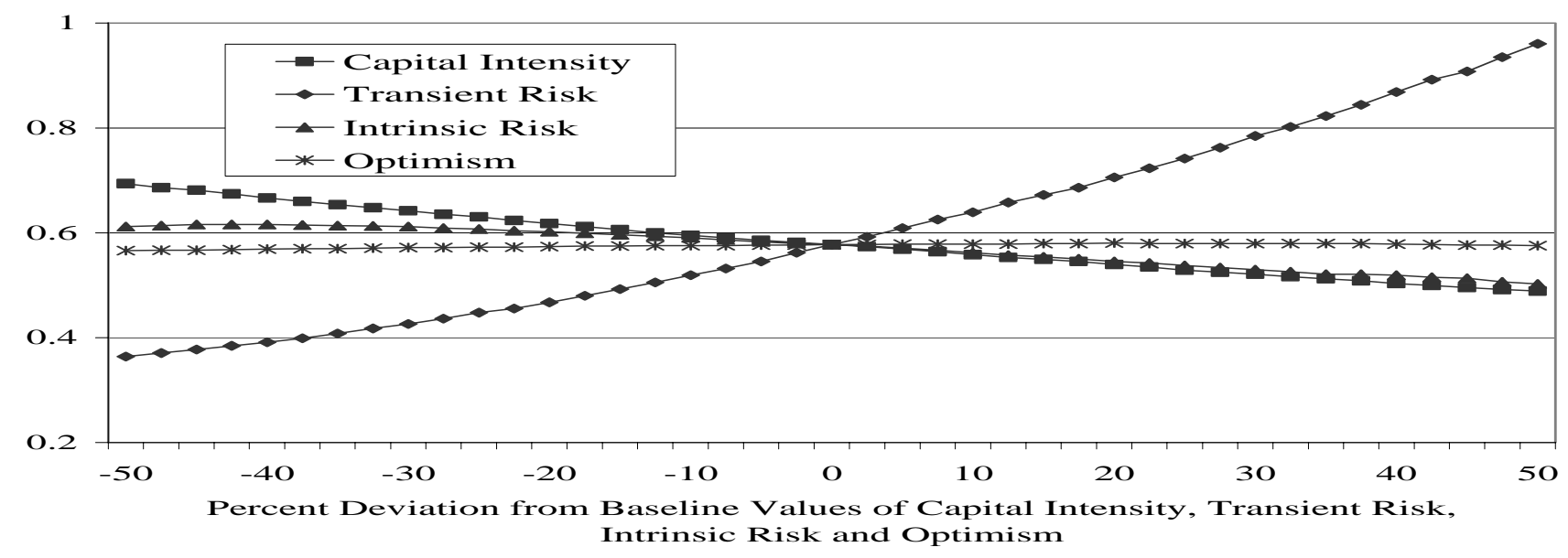

